\title{
Enabling Mechanical Separation of Enantiomers through Controlled Batch-wise Concomitant Crystallization: Digital Design and Experimental Validation
}

\author{
Andrew S. Dunn ${ }^{1,2}$, Botond Szilagyi ${ }^{3}$, Joop H. ter Horst ${ }^{2,4, *}$, Zoltan K. Nagy ${ }^{3,5, *}$ \\ ${ }^{l}$ EPSRC Doctoral Training Centre in Continuous Manufacturing and Crystallisation \\ embedded in the Future Manufacturing Research Hub for Continuous Manufacture and \\ Advanced Crystallisation (CMAC), Technology and Innovation Centre, University of \\ Strathclyde, 99 George Street, Glasgow, G1 1RD, UK \\ ${ }^{2}$ Strathclyde Institute of Pharmacy and Biomedical Sciences (SIPBS), University of \\ Strathclyde, 161 Cathedral Street, Glasgow, G4 0RE, UK \\ ${ }^{3}$ Davidson School of Chemical Engineering, Purdue University, West Lafayette, Indiana \\ 47907, United States \\ ${ }^{4}$ EPSRC Future Manufacturing Research Hub for Continuous Manufacture and Advanced \\ Crystallisation (CMAC), Technology and Innovation Centre, University of Strathclyde, 99 \\ George Street, Glasgow, G1 1RD, UK \\ ${ }^{5}$ Department of Chemical Engineering, Loughborough University, Loughborough, LE11 \\ 3TU, UK
}

*Corresponding authors e-mail: $\quad \frac{\text { joop.terhorst@strath.ac.uk }}{\underline{\text { knnagy@purdue.edu }}}$

\begin{abstract}
In the pharmaceutical industry the separation of chiral molecules is important due to the different physiochemical properties that the enantiomers of a chiral drug possess. Therefore, resolution techniques are used to separate such enantiomers from one another. In particular, preferential crystallization is a common technique used to separate conglomerate forming compounds, due to its high selectivity. However, efficient separation of enantiomers in a batchwise preferential crystallization process through seeding with the preferred enantiomer alone is still inefficient since unwanted nucleation of the counter enantiomer is inevitable. Here we demonstrate a novel method for the separation of enantiomers for a conglomerate forming compound (asparagine monohydrate), by using mechanical separation by sieving after crystallization, whereby the separation is enabled by a designed bias in the crystal size distributions of each enantiomer. This bias is created by a concomitant crystallization of both enantiomers using optimized seeding and cooling profiles obtained from a population balance model. In this way, a high level of control is achieved over a batch-wise preferential crystallization process since the crystallization of both enantiomers is controlled. We show that through this separation method, material with impurity levels as low as $6 \mathrm{wt} \%$ can be obtained.
\end{abstract}


To our knowledge this is the first demonstration of modelling such a process to separate enantiomers of a conglomerate forming compound.

Keywords: Preferential Crystallization, Mechanical Separation, Concomitant Crystallization, Digital Design of Crystallization, Crystallization Control, Enantioseparation, Asparagine Monohydrate 


\section{INTRODUCTION}

The pharmaceutical industry requires the use of resolution processes in order to separate chiral molecules to obtain a homochiral product. ${ }^{1}$ The importance of the access to efficient resolution processes is clear from the physiochemical properties of chiral drug substances. There are rare cases where both enantiomers (mirror image forms) of a chiral molecule induce the desired pharmaceutical response. In other cases, one of the enantiomers has the desired pharmaceutical response while the other enantiomer is inactive. However, in the more serious case, the opposite enantiomer can induce a hazardous effect on the patient. ${ }^{2}$ Such is the case of Naproxen: the $S$ enantiomer produces the desired anti-inflammatory response, whereas its mirror image $R$ enantiomer causes liver poisoning. ${ }^{3}$

There are a number of process techniques available to separate enantiomers, including deracemization ${ }^{4-6}$ and chromatography ${ }^{7-9}$ techniques. A common resolution technique used in industry in preferential crystallization (PC). In a PC process, a racemic or enantiomerically enriched solution is seeded with the preferred enantiomer, thereby allowing this form to crystallize out of solution and grow. ${ }^{10}$ One aspect of the PC process which still causes problems, however, particularly in batch setups, is the unwanted primary nucleation of the counter enantiomer. ${ }^{11}$ As the preferred enantiomer crystallizes, the supersaturation with respect to the counter enantiomer remains constant or, in the base of cooling or evaporative crystallization processes, increases in time. If the process is run for too long, this unwanted form will eventually crystallize via primary nucleation, in which case the process must be stopped. The likelihood of this occurring increases with increasing supersaturation and batch time. The risk of unwanted nucleation of the counter enantiomer can be reduced by running the PC process in a coupled batch ${ }^{12-14}$ or continuous setups. ${ }^{15-17}$ Recently, we demonstrated a control strategy that can be implemented in each of these setups that rectifies any occurrences of unwanted nucleation of the counter enantiomer. ${ }^{16}$ Furthermore, additives can be used to inhibit the crystallization of the unwanted chiral form. ${ }^{18}$ However, the problem remains that the risk of unwanted counter enantiomer nucleation is ever present.

It is important, therefore, to investigate other methods to separate enantiomers, for example, through mechanical separation. Mastai et al. ${ }^{19}$ exploited the difference in densities between racemic and enantiopure crystals of a racemic forming compound based on a density gradient method to obtain up to $70 \%$ separation of forms. However, such exploitation of solidstate properties cannot be used for conglomerate forming compounds since they are equal for both enantiomers. Furthermore, manipulation of the size and shape is difficult to control for 
only one enantiomer without affecting the other. Therefore, manipulation of the crystal size distributions (CSDs) for each enantiomer can be used via seeded batch-wise concomitant crystallization. Recently, purification by mechanical separation based on particle size has been demonstrated for a binary eutectic mixture of fluorene and fluorenone by Zhang et al. ${ }^{20}$ Doki et al. ${ }^{21}$ described a process to separate D- and L- crystals of asparagine based on their product crystal size. However, the use of additives was employed to delay the crystallization of Lasparagine such that the product crystal size distribution was bimodal. A patent by Maillard et al. ${ }^{22}$ also describes the process of separating enantiomers by their product crystal size by concomitant crystallization without the use of additives. However, none of the aforementioned works have utilized mathematical modelling to optimize the process.

In this paper we demonstrate a model-based digital design for the mechanical separation strategy for a conglomerate forming system of enantiomers to optimize the process of creating a bias in the crystal size distribution of each enantiomer. This strategy removes the risk of unwanted primary nucleation of the counter enantiomer by obtaining control of the crystallization of both enantiomers simultaneously. As a model system the separation of DLasparagine monohydrate (DL-asn) and the corresponding enantiomers L-asparagine monohydrate (L-asn) and D-asparagine monohydrate (D-asn) are used. First, we describe the formulation of a dynamic crystallization model of the process by means of population balance equations (PBEs), which is then used in the digital design of the system by performing a twolevel model-based optimization in order to obtain optimum crystallization conditions for experimentation. Finally, we experimentally demonstrate the use of the mechanical separation method using the results of the model in order to separate enantiomers of the conglomerate forming compound asparagine monohydrate.

\section{MODEL DEVELOPMENT AND NUMERICAL SOLUTION}

Population balance models (PBMs) are a widely used way to model crystallization processes. The population balance equation (PBE) describes the evolution of the solid phase within the crystallizer. The PBM is constructed by one or more PBEs, which are partial differential equations (PDEs) that describe the change in the number density function $n$ in time. The number density gives the number of particles per unit of volume within the $[x, x+d x]$ size interval at time $t$. In a PC process for a conglomerate forming compound, the solute molecules of one enantiomer will only crystallize on the seeds of this particular enantiomer. Hence, in a PC process for a conglomerate systems two populations can be distinguished: the population 
of L- and D- enantiomer crystals, for which the number densities are denoted by $n_{L}(x, t)$ and $n_{D}(x, t)$, respectively.

In this study, we assume a growth-only concomitant crystallization process with negligible agglomeration and breakage of particles. In such crystallization process, the number density of crystals remains constant and the seed crystals grow during the process. This is a good approximation if the crystallization process is kept within the metastable zone in order to avoid nucleation. Furthermore, the agitation intensity must be high enough to keep the system well-mixed and minimize the agglomeration, but at the same time mild enough to prevent crystal breakage. This process will be referred to hereafter as a well-controlled crystallization. The one-dimensional growth only PBE for such a well-controlled batch-wise cooling crystallization, can be given by:

$$
\frac{\partial n_{I}(x, t)}{\partial t}+G_{I} \frac{\partial n_{I}(x, t)}{\partial x}=0, \quad I=L, D
$$

where the initial and boundary conditions are given as,

$$
\begin{aligned}
& n_{I}(x, 0)=n_{I, 0}(x) \\
& n_{I}(x \rightarrow \infty, t)=0
\end{aligned}
$$

The initial condition $n_{I, 0}(x)$ gives the seed distribution of L-asn and D-asn whereas the boundary condition states that the crystals have finite size. In Eq. (1) $G_{I}$ is the size independent growth rate of enantiomer $I$ in $\mu \mathrm{m} / \mathrm{s}$, which is modeled by the power law equation:

$$
G_{I}=k_{g}\left(\frac{C_{I}}{C_{I, \text { sat }}}-1\right)^{g}
$$

where $k_{g}$ and $g$ are kinetic parameters, which are independent of enantiomeric form, $C_{I}$ is the solution concentration of the L- and D- enantiomers $\left(\mathrm{kg} / \mathrm{m}^{3}\right.$ of solvent) and $C_{I, \text { sat }}$ is the saturation concentration of an enantiomer. For the solubility description of asparagine the model equation given by Petruševska-Seebach et al. is used: ${ }^{23}$ 


$$
w_{e q}^{L}=p_{1} \exp \left(p_{2} \mathrm{~T}\right)+p_{3} w^{D}
$$

where $w_{e q}^{L}$ denotes the solubility of L-asn in terms of weight fraction as a function of temperature $\left({ }^{\circ} \mathrm{C}\right)$ and counter enantiomer weight fraction $w^{D}$. The weight fraction-based concentration can easily be recalculated to match the unit of concentration for $C_{I}$ based on the following equation:

$$
C_{L}=\rho \frac{w^{L}}{1-\left(w^{L}+w^{D}\right)}
$$

where $\rho$ is the solution density, expressed in $\mathrm{kg} / \mathrm{m}^{3}$. According to the solubility equation, the L-asn solubility depends on temperature and the concentration of D-asn. A similar equation is applicable for a D-asn solubility calculation. Hence, the dynamics of the two populations are not independent of each-other being linked through the solubility expression. However, based on the solubility expression this effect is small. The change in concentration inside the crystallizer as a function of time is given by the solute mass balance, which is written in the form of an ordinary differential equation (ODE):

$$
\frac{d C_{I}}{d t}=-3 \rho_{c} k_{v} G_{I} 10^{-18} \int_{0}^{\infty} L^{2} n_{I}(x, t) d x, \quad I=\mathrm{L}, \mathrm{D}
$$

where $\rho_{c}$ is the crystal density $\left(\mathrm{kg} / \mathrm{m}^{3}\right), k_{v}$ is the volume shape factor and $10^{-18}$ is a factor that transforms the particle size units from $\mu \mathrm{m}^{3}$ to $\mathrm{m}^{3}$. Here we assumed that the L-asn and $\mathrm{D}$-asn crystals have identical densities and shapes. The initial conditions of the mass balance equations are the initial concentration: $C_{I}(t=0)=C_{I, 0}$.

\subsection{Numerical solution of the model-equations}

Various techniques have been developed to solve PBEs. One of the more popular methods is to use the standard method of moments (SMOM) ${ }^{24}$ and its quadrature based extensions. ${ }^{25}$ This enables the exact calculation of moments of the distribution and the concentration however it fails to calculate the full CSD. Among the full PBE solvers the Method of Characteristics (MOC) is known to be the most accurate as it completely removes the convective term from the hyperbolic PBE. ${ }^{26}$ For crystallization process simulation MOC can be numerically inaccurate because of the mass balance evaluation of Eq. (5), unless a very fine discretization 
is applied, which in turn causes it to become numerically inefficient. In this work, SMOM is employed for concentration calculation, and the combined SMOM and MOC for the accurate and efficient full CSD calculation. These are well known techniques ${ }^{27}$ and thus only a brief description is provided here.

\subsubsection{SMOM for solute concentration calculation}

The SMOM relies on the moment transformation rule, given by the following equation:

$$
\mu_{I, i}=\int_{0}^{\infty} L^{i} n_{I}(x, t) d L
$$

Applying the moment transformation to the PBE in Eq. (1) gives the moment equations system:

$$
\begin{aligned}
& \frac{d \mu_{I, 0}}{d t}=0 \\
& \frac{d \mu_{I, i}}{d t}=i G_{I} \mu_{I, i-1} \quad i=1,2,3
\end{aligned}
$$

with the moment transformation, the mass balance in Eq. 5 becomes:

$$
\frac{d C_{I}}{d t}=-3 \rho_{c} k_{v} G_{I} \mu_{I, 2} 10^{-18}
$$

Considering the first four moments of each enantiomer distributions, the system of moment equations for the two populations (Eq. 7) with the corresponding mass balances (Eq. 8) generates ten ordinary differential equations (ODEs) which consist of the closed model of the well-controlled PC process.

\subsubsection{Combined SMOM and MOC for full CSD calculation}

The MOC is a well-suited solution method of the PBE shown in Eq. (1) for full CSD calculation. $^{23,28,29}$ Through the MOC curves are defined in the $(x-t)$ plane, which reduces the PBE to a system of ODEs. The $(x-t)$ plane can be expressed in a parametric form by $x=x(Z)$ and $t=t(Z)$, where the parameter $Z$ gives the measure of distance along the characteristic curve. The number density $n_{I}$ can be redefined as: 


$$
n_{I}(x, t)=n_{I}(x(Z), t(Z))
$$

Applying the chain rule on we obtain:

$$
\frac{d n_{I}(x, t)}{d Z}=\frac{d t}{d Z} \frac{\partial n_{I}(x, t)}{\partial t}+\frac{d x}{d Z} \frac{\partial n_{I}(x, t)}{\partial x}
$$

Comparing the coefficients of Eqs. 1 and 10 it can be concluded that:

$$
\begin{aligned}
& \frac{d t}{d Z}=1 \rightarrow d t=d Z \\
& \frac{d x}{d Z}=G_{I} \leftrightarrow \frac{d x}{d t}=G_{I} \\
& \frac{d n_{I}(x, t)}{d Z}=0 \leftrightarrow \frac{d n_{I}(x, t)}{d t}=0
\end{aligned}
$$

with initial conditions, corresponding to $t=Z=0, x=x_{0}$ and $n_{I}(x, 0)=n_{I, 0}(x)$. Eq. (13) expresses that the number of crystals belonging to the characteristic lines remain unchanged throughout the process. To obtain the dynamic evolution of the crystal size distribution, $n_{I}(x, 0)$, Eq. (12), with prescribed growth expressions, can be integrated repeatedly for different initial values $\left[x_{0}\right]$. The mass balance Eq. (5) can be evaluated using the MOC based CSD. However, for accurate mass balance calculation a high number of characteristic lines have to be used i.e. Eq. (12) is solved for many different $\left[x_{0}\right]$ values, which degrades the overall computational efficiency. As an efficient workaround, the MOC can be effectively combined with the SMOM by first applying the SMOM solution, which provides the accurate concentration profile as an input for the growth rate calculation within the MOC. This decouples the accuracy of CSD dynamics from the MOC discretization coarseness and hence, the computational time. ${ }^{23}$

\section{DIGITAL DESIGN VIA MODEL-BASED OPTIMIZATION}

The digital design of the PC system was carried out by applying model-based optimization for the determination of optimum process conditions subject to the set of constraints and 
objectives. ${ }^{30,31}$ The batch PC process to be optimized in which a racemic solution of enantiomers is seeded with crystals consisting of both chiral forms but with different crystal sizes and seed loadings. In this way, we can create a bias in the product crystal size of each enantiomer and thus separate these enantiomer crystals by means of a mechanical process such as sieving, sedimentation, centrifugation and others. In this section, we briefly present the developed novel optimization framework applied in this study. A schematic representation of the optimization procedure is shown in Figure 1.

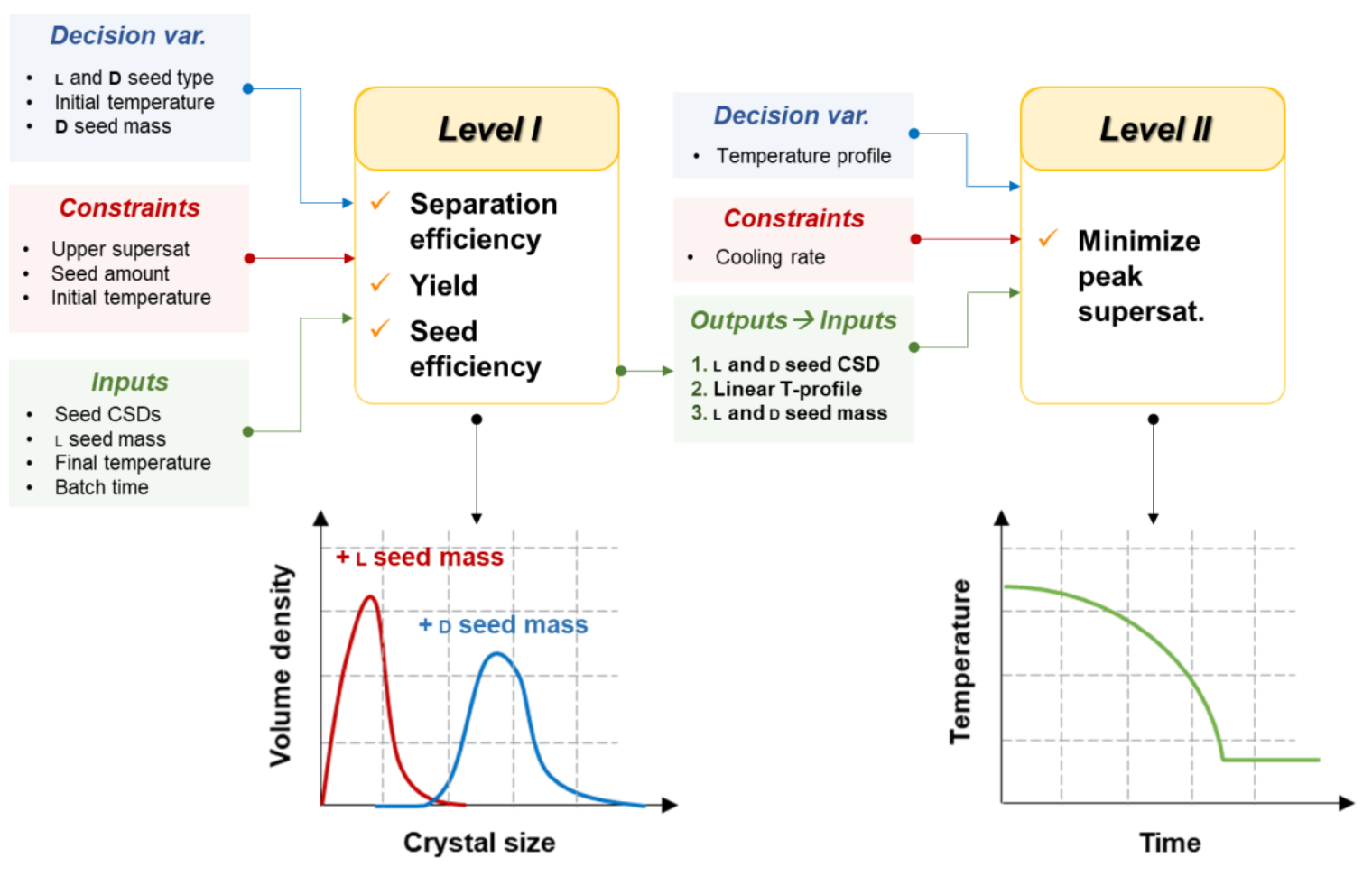

Figure 1. Schematic representation of the model-based optimization framework for the mechanical separation of enantiomers of a conglomerate forming compound. Input parameters, decision variables and constraints are applied to a Level 1 optimization to optimize the separation efficiency, yield and seed efficiency of the process. This initial optimization generates outputs of initial seed CSD and seed loading for each enantiomer, as well as an optimized linear cooling profile. These Level 1 optimization outputs are then used as inputs alongside additional decision variables and constraints for a Level 2 optimization, which generates the optimum dynamic cooling profiles in order to minimize the peak supersaturation achieved during the process, which could lead to contamination through unwanted secondary nucleation. 
It is important to understand that the product CSD of a growth-only batch crystallization process is fully determined by the seed CSD and operating supersaturation domain. In this context, one might have the impression that for the purpose of this work it is sufficient to use the traditional equations for the seed recipe design (Eq. (24)). There are two problems with this assumption; first, this is valid if the crystallizer is operated within the metastable zone assuming all crystals are identical in size. This leads to the second issue; to ensure growth-only conditions, low supersaturation must be applied throughout the process, leading to long batch times. To improve the performance, dynamic simulation-based optimizations are required that can operate directly on nonlinear constraints, such as maximum allowed supersaturations, as well as various combinations of objectives.

\subsection{The Objective}

We aim to maximize three sub-objectives, namely, the separation efficiency $e$, the yield $y$ and the seed efficiency $s$, combined in one average objective, using a weighted sum of these:

$$
O=O_{e}+w_{1} O_{y}+w_{2} O_{s e}
$$

where $w_{1}$ and $w_{2}$ are weight factors that control the relative importance of each sub-objective. Assuming complete physical separation by the sieves, which is a reasonable approximation for crystals having closely spherical shape, the separation efficiency is defined as the fraction of the crystallized product volume that is present in enantiopure sieve fractions:

$$
O_{e}=\sum_{i=1}^{N} \varepsilon_{i} \Phi_{i}
$$

where $i$ stands for the $i^{\text {th }}$ sieve fraction, $\varepsilon_{i}$ is a binary variable that characterizes the separation in the $i^{t h}$ sieve fraction having the value $\varepsilon_{i}=1$ if the solid enantiomer excess $E_{c r}$ exceeds a threshold value in the given sieve fraction, and it is 0 otherwise:

$$
\varepsilon_{i}=\left\{\begin{array}{c}
1, \text { if } \int_{L_{b, i}}^{U_{b, i}} \frac{\left|n_{L, v}\left(x, t_{f}\right)-n_{D, v}\left(x, t_{f}\right)\right|}{\left[n_{L, v}\left(x, t_{f}\right)+n_{D . v}\left(x, t_{f}\right)\right]} d x>E_{c} \\
0, \text { otherwise }
\end{array}\right.
$$


where $n_{L, v}$ and $n_{D, v}$ are the volume based CSDs of L- and D- enantiomers, respectively and $t_{f}$ is the final time. The sizes $L_{b, i}$ and $U_{b, i}$ are the lower and upper size limit of the $i^{\text {th }}$ sieve fraction. The volume fraction of the crystals in the given sieve fraction, $\Phi_{i}$ is given by:

$$
\Phi_{i}=\frac{\int_{L_{b, i}}^{U_{b, i}}\left[n_{L, v}\left(x, t_{f}\right)+n_{D, v}\left(x, t_{f}\right)\right] d L}{\mu_{L, 3}\left(t_{f}\right)+\mu_{D, 3}\left(t_{f}\right)}
$$

It is assumed that the solution is in equilibrium i.e. there is no supersaturation at the beginning and the end of crystallization process. The yield, defined as the fraction of solute crystallized out, is expressed in terms of solubilities at the corresponding temperatures:

$$
O_{y}=\frac{\left[C_{L, s a t}\left(T_{i}\right)-C_{L, s a t}\left(T_{f}\right)\right]+\left[C_{D, s a t}\left(T_{i}\right)-C_{D, s a t}\left(T_{f}\right)\right]}{C_{L, s a t}\left(T_{i}\right)+C_{D, s a t}\left(T_{i}\right)}
$$

where $T_{i}$ and $T_{f}$ denote the initial and final temperatures.

The seed efficiency sub-objective expresses the mass of product obtained from unit mass of seed:

$$
O_{s e}=\frac{\mu_{L, 3}\left(t_{f}\right)+\mu_{D, 3}\left(t_{f}\right)-\mu_{L, 3}\left(t_{i}\right)-\mu_{D, 3}\left(t_{i}\right)}{\mu_{L, 3}\left(t_{i}\right)+\mu_{D, 3}\left(t_{i}\right)}
$$

The introduction of seed efficiency term is necessary since the seeds are high quality crystals obtained from the product of previous batches, hence the seed production reduces the overall productivity. The weight factors of the optimization were set based on preliminary optimizations such to assign the highest relative importance to separation efficiency, whereas slightly lower and relatively equal importance to yield $\left(w_{1}=0.01\right)$ and seed efficiency $\left(w_{2}=\right.$ $0.05)$.

\subsection{The Decision Variables}

In order to simplify the optimization problem, the following quantities were given fixed values: batch time (with a certain equilibrium time after the final temperature is applied for de- 
supersaturation), final crystallization temperature and seed loading of L-asn crystals. L-asn seed loading is fixed to give some level of control over the optimization to the human operator.

The following continuous and discrete decision variables therefore still exist:

- D-asn seed loading (continuous),

- seed type given by a particular fraction (integer),

- initial temperature (continuous) and

- vector of temperatures that define the cooling profile (continuous)

In practice L-asn and D-asn seed crystals were obtained by sieving. There are no arbitrary sieve openings, therefore the seed distribution cannot be directly optimized. In contrast, different sieve fractions can be used as seeds. For example, one might apply 63-90 $\mu \mathrm{m}$ L-asn and 90-125 $\mu \mathrm{m}$ D-asn seed sieve fractions. Hence, the L-asn and D-asn seed type is an integer decision variable. The problem becomes a mixed integer nonlinear programming (MINLP).

\subsection{Constraints and Solution Techniques}

Two-level solution was applied for the optimization problem that considerably improves the chance of finding the global optimum. As Figure 1 presents, the first level optimization results in a solution of the MINLP maximization problem by an exhaustive search i.e. carrying out optimization for every possible combination of L-asn and D-asn sieve fractions, based on the following objective:

$$
\max _{T_{i}, \Phi_{D}, U_{L}, U_{D}}\left\{O_{1}\left(T_{i}, \Phi_{D}, U_{L}, U_{D}\right)=O_{e}+w_{1} O_{y}+w_{2} O_{s e}\right\}
$$

In this optimization the linear cooling profile is applied with a fixed final temperature. $T_{i}$ is the initial temperature (the final temperature is fixed at $20^{\circ} \mathrm{C}$ ), $\Phi_{D}$ is the volume fraction of D-asn seeds in the total suspension volume ( $\Phi$ of L-asn seeds is fixed at 0.0025 ) and $U_{L}$ and $U_{D}$ are the integer labels representing the sieve fractions for L-asn and D-asn seed types (seed sieve fractions). For example, $U_{L}=1$ for the smallest available sieve fraction of L-asn seeds, $U_{L}=2$ for the second smallest sieve fraction etc. The following constraints for the crystallization process were applied: 


$$
\begin{aligned}
& \max \left\{C_{L}(t), C_{D}(t)\right\} \leq C_{M S}(T(t)) \\
& T_{i, \min } \leq T_{i} \leq T_{i, \max } \\
& \Phi_{D, \min } \leq \Phi_{D} \leq \Phi_{D, \max }
\end{aligned}
$$

The first constraint avoids conditions for secondary nucleation or other nucleation processes to occur by limiting the process to operate within the metastable zone by avoiding the concentration to exceed the metastable limit. Secondary nucleation would generate new crystals to act as seeds which leads to a loss of control over the particle size distributions. $C_{M S}$ is the assumed secondary nucleation curve (see supporting information for the determination of $C_{M S}$ ). The second constraint limits initial temperature $T_{s a t}$, that also affects the initial saturation concentration. This allows for a sufficient amount of crystallization to occur during the process with wasting vast amounts of material by starting at significantly high solution concentrations. The third constraint limits the volume fraction of D-asn seed crystals.

The second optimization $\mathrm{O}_{2}$ was carried out to find the optimal temperature profile. The temperature profile is defined as a vector of temperatures $\left(T_{V}\right)$ in the intermediate time moments corresponding to the elements of the time vector $\left(t_{V}\right) . t_{V}$ is the vector of evenly spaced time moments between the initial and final process time. To improve the CPU time of optimization, two, successive optimizations were solved: in the first optimization $t_{V}$ is set to 10 , whereas the second optimization $t_{V}$ is refined to 20 but the initial $T_{V}$ was obtained by interpolating the solution of the first optimization to this finer time grid. Therefore, the second optimization involving more decision variables is a warm-start optimization, which has reduced CPU time compared to its cold-start counterpart. ${ }^{32}$ The objective $\mathrm{O}_{2}$ is the minimization of peak supersaturation by re-adjusting the temperature profile between the fixed initial and final temperatures, which further reduces the chance of unwanted secondary nucleation:

$$
O_{2}\left(T_{V}\right)=\max \left\{S_{L}\left(t_{v}\right), S_{D}\left(t_{v}\right)\right\}
$$

where $S_{L}\left(t_{v}\right)$ and $S_{D}\left(t_{v}\right)$ denote the vectors of supersaturation throughout the batch for both enantiomers. The second optimization has the constraints: 


$$
c_{r, \min } \leq c_{r}=-\frac{\Delta T}{\Delta t} \leq c_{r, \max }
$$

where $c_{r}$ is the cooling rate.

The kinetic and process parameters used in the simulation case study are listed in Table 1. The solubility equations and growth kinetics are directly taken from literature ${ }^{23,33}$.

Table 1. Model parameters applied in this study, taken from the literature. ${ }^{23,33}$

\begin{tabular}{lccc}
\hline Parameter & Symbol & Value & Units \\
\hline Growth rate constant & $k_{g}$ & 5.044 & $\mu \mathrm{m} / \mathrm{s}$ \\
Growth rate supersaturation exponent & $g$ & 1.48 & - \\
Crystal volume shape factor & $k_{v}$ & 1.87 & - \\
Crystal density & $\rho_{c}$ & $1.554 \times 10^{3}$ & $\mathrm{~kg} / \mathrm{m}^{3}$ \\
Solubility parameter & $p_{1}$ & $9.2 \times 10^{-3}$ & - \\
Solubility parameter & $p_{2}$ & $4.43 \times 10^{-2}$ & - \\
Solubility parameter & $p_{3}$ & $3.048 \times 10^{-2}$ & - \\
\hline
\end{tabular}

\section{EXPERIMENTAL}

\subsection{Materials and Methods}

DL-asparagine monohydrate (DL-asn) and L-asparagine monohydrate (L-asn) and D-asparagine monohydrate (D-asn) were purchased from Sigma-Aldrich (Germany). Seed crystals of L-asn were prepared through subsequent sieving using the Fritsch Analysette 3 Pro sieve shaker to obtain the desired sieve fractions described in Table 2. De-ionised water, purified by Milli-Q gradient system from Milipore SAS (France), was used as a solvent.

Chiral HPLC analysis was carried out using an Agilent HPLC instrument to determine enantiomeric excess of the solution and recovered solid product. Solid samples obtained from the filtered slurry were dissolved in water at a concentration of $1 \mathrm{mg} / \mathrm{mL}$ solvent. A CHIROBIOTIC T column (Astec, $150 \times 4.6 \mathrm{~mm}$ with $5 \mu \mathrm{m}$ particle size) was used at $25.0^{\circ} \mathrm{C}$ with a 70/30 methanol/water (v/v) mobile phase (flow rate of $0.5 \mathrm{~mL} / \mathrm{min}$ ) with UV detection at $205 \mathrm{~nm}$. The injection volume was $5 \mu \mathrm{L}$.

Experimental determination of the L-asn and D-asn seed CSDs was carried out using a Malvern MasterSizer3000 fitted with Hydro SM dispersion unit. Dry seeds crystals from each 
sieve fraction were dispersed in IPA and introduced into the flow cell of the Mastersizer unit via a circulation system for CSD measurement by laser diffraction. Five representative measurements of each sample were collected, and the average values are reported here.

\subsection{Concomitant Crystallization Experiments}

Separation experiments were performed in a Mettler Toledo OptiMax ${ }^{\mathrm{TM}}$ workstation of $1 \mathrm{~L}$ capacity, equipped with an in-line Hastelloy® Pt100 temperature sensor. The system was operated and controlled using iControl V5.2 software. For in-situ particle tracking a MettlerToledo FBRM probe (G400 series) was inserted into the reactor and added to the workstation with iC FBRM V4.3 incorporated into the iControl software. For in-situ particle imaging a Mettler-Toledo Particle Vision and Measurement (PVM) was inserted into the reactor and added to the workstation with iC PVM V7.0 incorporated into the iC Control Software. DL-asn was added to water in the OptiMax ${ }^{\mathrm{TM}}$ reactor such that an overall composition required as dictated by Table 2 was obtained. The solution was heated to $10^{\circ} \mathrm{C}$ above the saturation temperature $T_{s}$ to ensure all particles dissolved and a clear solution was obtained. The temperature was then reduced to $T_{s}$ and held for 10 minutes. The optimized temperature profile was then started. Two optimized cooling times were used; 5-hour and 3-hour, each of which had an additional 2-hour equilibration time at the final crystallization temperature of $20^{\circ} \mathrm{C}$. An example of one of the optimized cooling profiles can be seen in Figure 4 in Section 5.1. At this point seeds of both L-asn and D-asn were added in accordance with Table 2. Experiments were carried out using different volume fraction seed loadings of L-asn and D-asn seeds and different seed sizes of each.

Table 2. Optimized experimental parameters for the experimental demonstration of the separation strategy. The seed volume fraction $\Phi$ is define as the ratio between the volume of seed of L-asn or D-asn and the total volume of the suspension.

\begin{tabular}{cccccccccc}
\hline & & \multicolumn{3}{c}{ L-asn } & \multicolumn{5}{c}{ D-asn } \\
\cline { 3 - 7 } Experiment & $T_{s}$ & Seed & Volume & Seed & Seed & Volume & Seed & Cooling \\
& {$\left[{ }^{\circ} \mathrm{C}\right]$} & Size & Fraction & Mass & Size & Fraction & Mass & Time \\
& & {$[\mu \mathrm{m}]$} & $\Phi_{\mathrm{L}}$ & {$[\mathrm{g}]$} & {$[\mu \mathrm{m}]$} & $\Phi_{\mathrm{D}}$ & {$[\mathrm{g}]$} & {$[\text { hours }]^{a}$} \\
\hline 1 & 44.8 & $63-90$ & 0.0017 & 1.33 & $180-$ & 0.0025 & 1.96 & 5 \\
& & & & & 250 & & &
\end{tabular}


90-

0.0010

$\overline{{ }^{a} \text { Cooling time refers to the number of hours between the initial and final temperatures and does }}$ not include the 2-hour equilibration time when the final temperature is reached

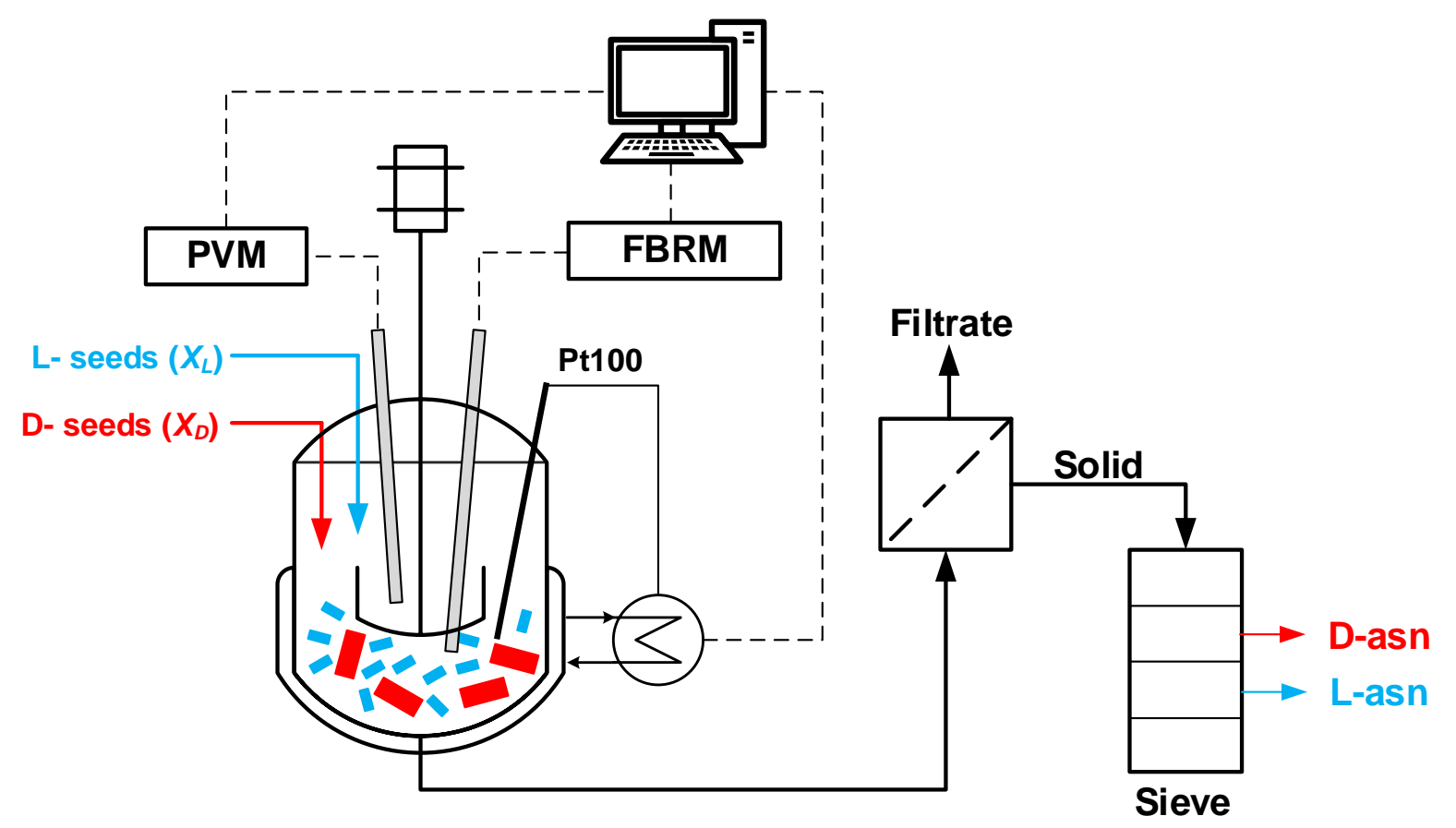

Figure 2. Batch PC setup for the mechanical separation of D-asn and $\mathrm{L}$-asn enantiomers. A $1 \mathrm{~L}$ Optimax reactor was used for the crystallization process, which was seeded with both D-asn and L-asn crystals as detailed in Table 2. Once the cooling profile was complete the suspension was collected, filtered and washed. Product crystals were left to dry in a vacuum oven at $25^{\circ} \mathrm{C}$ overnight before being sieved. Samples of product collected in each sieve fraction were collected for enantiomeric excess determination by chiral HPLC.

Once the process was complete, the suspension was removed from the vessel and filtered using the Buchner funnel. The filtrated solid was washed one with solvent (water) and three times with anti-solvent, isopropanol (IPA), and left to dry overnight in a vacuum oven. For each 
wash, solvent was used at room temperature and enough solvent was added to the Buchner funnel to just cover the filtrate cake. It was found during experimentation that filtration and washing of the product crystals is extremely important; in preliminary experiments, no optimized washing procedure was in place and after drying the product crystals were heavily agglomerated. Therefore, the separation results obtained for these experiments were quite poor. However, once an improved washing regime was established, crystals were observed to be unagglomerated and free flowing. After drying the product was sieved using a Fritsch Analysette 3 Pro sieve shaker. Each sieved fraction was tared and weighed after sieving to determine the mass of product collected in each fraction. Finally, solid samples from each sieved fraction were taken for HPLC analysis to determine the solid enantiomeric excess. Product collected in a particular sieve fraction was considered impure if the enantiomeric excess obtained was less than $80 \%$. A schematic of the experimental setup and process procedure is shown in Figure 2.

\section{RESULTS}

\subsection{Concomitant Crystallization Simulations}

The performed simulation case study demonstrates the capabilities of the developed two-level optimization strategy. The first level optimization determines the optimum input seed size distributions and seed volume fractions $\Phi_{\mathrm{L}}$ and $\Phi_{\mathrm{D}}$ for L-asn and D-asn, respectively. The second level optimization then optimizes the cooling profile to minimize the peak supersaturation in order to avoid unwanted nucleation during the process. Four log-normal seed distributions are chosen for the L-asn and D-asn with volume based average size of 100 (CSD1), 150 (CSD2), 200 (CSD3) and $250 \mu \mathrm{m}$ (CSD4), which are plotted in Figure 3. This translates to 16 possible L-asn-D-asn seed pair CSD combinations. Carrying out an exhaustive search for this problem generate 16 Level 1 optimizations, which, given the efficient solver implementation does not take longer than a few hours, depending on the given machine configuration. Therefore, the computational time does not require MINLP solvers, and the chance of finding the global optimum is improved by the exhaustive search. In this simulation case study there are overlapping L-asn and D-asn combinations, but these are not necessarily symmetric because of the potentially different seed loadings. 


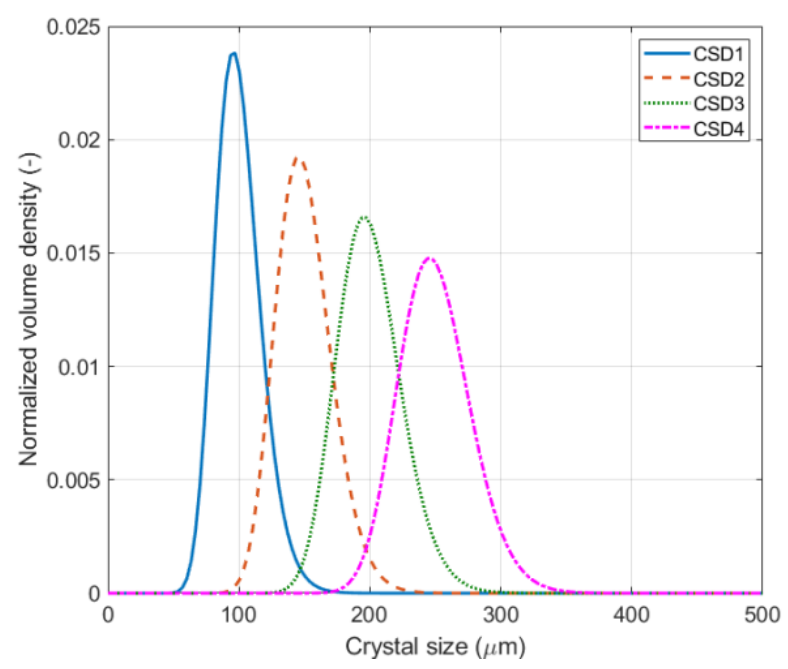

Figure 3. Seed distributions $\left(n_{0}\right)$ for both L-asn and D-asn in the simulation case study.

Table 3 lists the conditions and constraints of the process optimizations. In the simulation case study, the batch cooling time was set to 5 hours. However, in the experiments conducted both 3 and 5-hour batch cooling times were applied. The constraints for the initial temperature were set to lower and upper boundaries of 35 and $45^{\circ} \mathrm{C}$, respectively, whereas the final temperature was fixed. The maximum cooling rate was set such as to be well within the cooling capacity of the experimental system. L-asn seed volume fraction $\Phi_{\mathrm{L}}$ and the critical enantiomer excess $E_{c}$ (i.e. a product specification of $99 \%$ ) were set based on previous experimental work. ${ }^{16}$ D-asn seed volume fraction limits were defined as a symmetrical interval around the L-asn seed volume fraction.

Table 3. Parameters and constraints for optimization of the concomitant crystallization process

\begin{tabular}{lccc}
\hline Parameter & Symbol & Value & Units \\
\hline Batch time & $t_{b}$ & $5^{*}$ & $\mathrm{~h}$ \\
Equilibration time & $t_{e}$ & 2 & $\mathrm{~h}$ \\
Initial temperature & $T_{i}$ & $35-45$ & ${ }^{\circ} \mathrm{C}$ \\
Final temperature & $T_{f}$ & 20 & ${ }^{\circ} \mathrm{C}$ \\
L-asn seed volume fraction & $\Phi_{L}$ & 0.0025 & - \\
D-asn seed volume fraction & $\Phi_{D}$ & $0.001-0.005$ & - \\
Minimal cooling rate & $c_{r, \min }$ & 0.01 & ${ }^{\circ} \mathrm{C} / \mathrm{min}$ \\
Maximal cooling rate & $c_{r, \max }$ & 0.3 & ${ }^{\circ} \mathrm{C} / \mathrm{min}$ \\
Critical enantiomeric excess & $E_{c}$ & 0.99 & -
\end{tabular}


Sieve fractions boundaries

$\{20,45,63,90,125$,

$180,250,355,500\}$

$\mu \mathrm{m}$

*only batch times of 5 hour were used for simulation purposes

The Level 1 optimization results are presented in Table 4. As described in Table 3, a fixed Lasn seed volume fraction $\Phi_{\mathrm{L}}$ of 0.0025 was used for the simulation. The details of the results show the most optimum CSD combination for both L- (CSD4) and D-asn (CSD3) from the 16 possible combinations of all for CSDs in Figure 3. Naturally, these CSDs are optimal only in combination with the optimal initial temperature $\left(41.6^{\circ} \mathrm{C}\right)$ and D-asn seed volume fraction $\left(\Phi_{D}\right.$ $=0.0043)$ that were determined by the simulation in order to achieve $100 \%$ separation of enantiomers.

Table 4. Level 1 optimization output for the simulation-based case study for a fixed L-asn seed volume fraction $\Phi_{\mathrm{L}}$ of 0.0025 . The nomenclature for $\mathrm{L}$-asn and D-asn seeds is taken from Figure 3.

\begin{tabular}{cccc}
\hline $\begin{array}{c}\text { L-asn seed } \\
\text { distribution } n_{0}\end{array}$ & $\begin{array}{c}\text { D-asn seed } \\
\text { distribution } n_{0}\end{array}$ & $\begin{array}{c}\text { D-asn Volume } \\
\text { Fraction } \Phi_{D}\end{array}$ & $T_{i}\left({ }^{\circ} \mathrm{C}\right)$ \\
\hline CSD4 & CSD3 & 0.00435 & 41.6 \\
\hline
\end{tabular}

Figure $4 \mathrm{a}$ represents the normalized volume densities of the product crystal populations obtained in the two-level optimization together with the experimentally available sieve fraction boundaries. Crystals of D-asn would be collected in the $355-500 \mu \mathrm{m}$ sieve, with a very small portion collected in the $250-355 \mu \mathrm{m}$ sieve whilst the L-asn crystals would be collected in the 500-710 $\mu \mathrm{m}$ sieve fraction. Hence, the simulation suggests that perfect separation of the enantiomer crystals is achieved using the optimized crystallization conditions using the 500 $\mu \mathrm{m}$ sieve fraction. The level 1 optimized supersaturation of each enantiomer throughout the process is plotted in Figure 4b. Due to the constraint on the concentration (Eq. 21) in the Level 1 optimization, the supersaturation profile during the crystallization nicely stays within the metastable zone.

The Level 2 optimization minimizes the peak supersaturation by optimizing the temperature profile within the set constraints of cooling rate and process time. Figure $4 \mathrm{c}$ illustrates that the operating trajectories in the phase diagram are well within the (assumed) secondary nucleation metastable zone. Therefore, the probability of unwanted secondary 
nucleation is minimized in the Level 2 optimization. After addition of the seeds, the optimized temperature cooling profile (Figure 4d) has parabolic shape, which is known to be the most effective for avoiding unwanted nucleation, especially likely at the start of the batch, in seeded cooling crystallization. ${ }^{34}$ The peak supersaturation (which, in this case is for L-asn) plateaus at $\sim 7.8 \times 10^{-3} \mathrm{~g} / \mathrm{g}$ solvent between $10-90$ minutes of the process. The peak supersaturation in Level 1 optimization was $\sim 9.2 \times 10^{-3} \mathrm{~g} / \mathrm{g}$ solvent, therefore, the Level 2 optimization reduced the peak supersaturation in the system by $\sim 15 \%$ through small adjustments in the temperature profile, and hence provides a more robust process. The results also show that the optimization-based design can determine the optimal seed volume fraction of the two enantiomers and the optimal operating trajectory that can lead to complete separation of the product CSDs even if the seed CSDs of the two enantiomers have relatively large overlap.

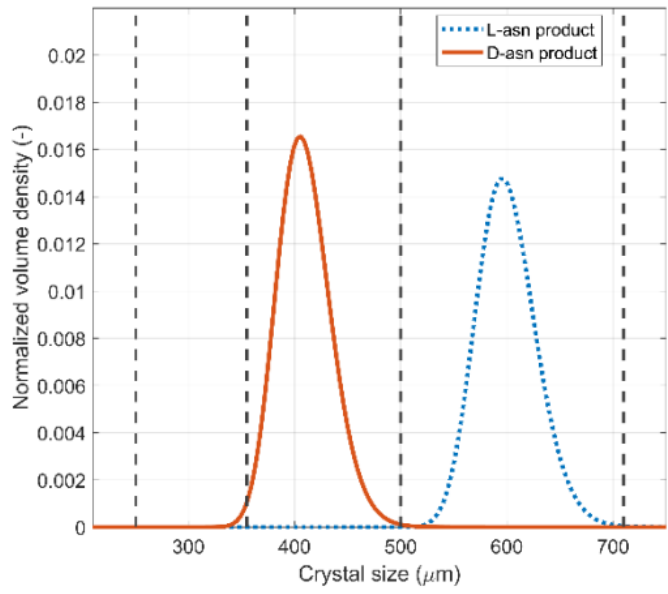

(a)

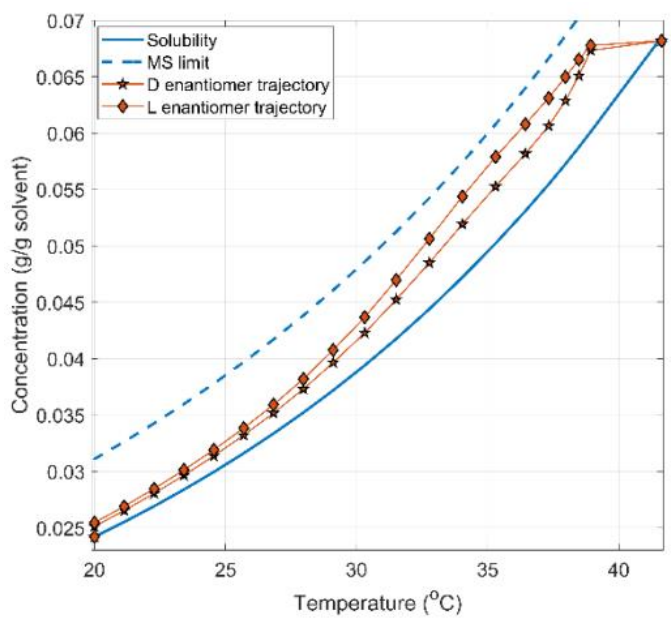

(c)

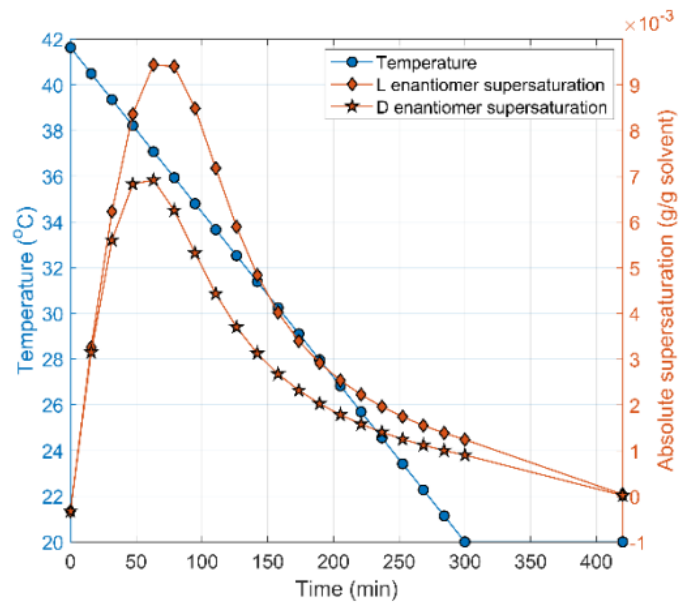

(b)

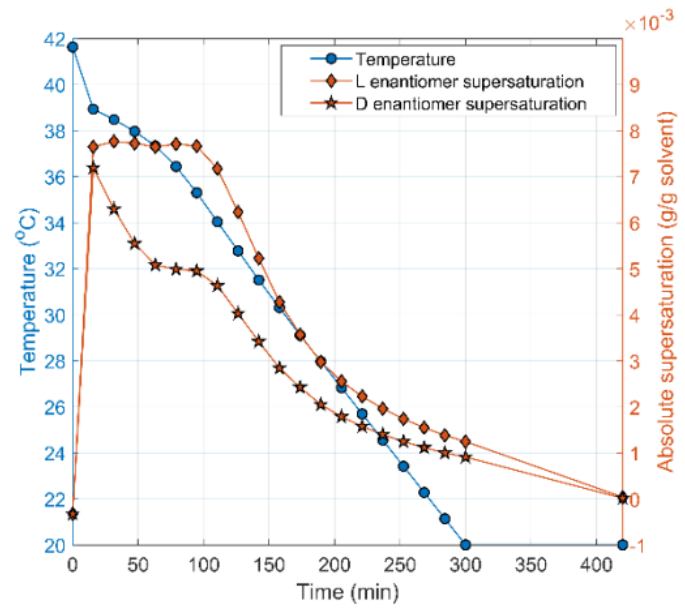

(d) 
Figure 4. Level 1 ( $a$ and b) and Level 2 (c and d) optimization results for the simulation with CSD3 seeds for D-asn and CSD4 seeds for L-asn: (a) the optimized product distributions and available sieve fraction boundaries, (b) optimized linear cooling profile and the resulting supersaturations for the L- and D- enantiomers, (c) phase diagram of the Level 2 optimized process and (d) The optimized temperature profile and the resulting supersaturations for the Lasn and D-asn after the level 2 optimization. The seeds are added at $t=0$.

\subsection{Parametric study and in silico design for separation efficiency}

According to the case study in Section 5.1, the developed optimization framework is suitable for calculating the optimal process conditions for efficient concomitant PC process design. However, from process understanding point of view, the optimum operation represents only that in a narrow slice of the operating space. A parametric study was carried out to analyze the process in a substantially broader space.

Figure 5 presents the weight fraction of enantiopure product obtained by sieving with the sieve fractions defined in Table 3. The initial temperature and seed CSDs were set based on the optimization results in Table 4 in order to obtain results comparable to the optimization section. Figure 5 shows the surface plots for the achievable separation performance by sieving as a function of seed loading volume fractions of both enantiomers. The yellow region in the top right shows seed loading combination that can achieve 100\% separation of both enantiomer product crystals. The dark blue region, in contrast, shows seed loading combinations of $0 \%$ separation i.e. complete overlap of the product CSDs of both enantiomers. The red star close to the boundary of $100 \%$ performance shows indicates the optimum solution point used in this work. From a mathematical perspective, having the solution close to the boundary works well. However, from a practical perspective it is possible to easily miss the product specifications, as only a small error in the implementations might significantly degrade the separation efficiency. However, robust optimization can be applied in future to keep some distance from these critical boundaries, for example by considering the effects of various experimental related uncertainties. In Figure 5a the critical enantiomeric excess was set to $90 \%$, while the critical enantiomeric excess for Figure 5b was set to $99 \%$. According to the results, by decreasing the product purity specifications, the operating space in which enantiopure product can be obtained increases, meaning that the process is easier to control (but with the disadvantage of losing some purity). As the rest of the process parameters of these simulations were set based on the optimization results, the seed loadings corresponding to the optimal values on the surface of Figure $5 \mathrm{~b}$ represent the optimum solution. This parametric study also reveals that at the given 
initial temperature and seed CSDs there are many other seed loadings that yield in $100 \%$ separation. This leaves room for the further optimization of secondary objectives, such as yield and seed efficiency (which are optimized in this work, however, they were not measured experimentally since they are fully defined by the seed mass and temperature range of the experiments). In the context of optimization, it must be highlighted that these surfaces do not consider the supersaturation and other constraints (Eq. 21), therefore some parts of the space might be infeasible for the optimization.
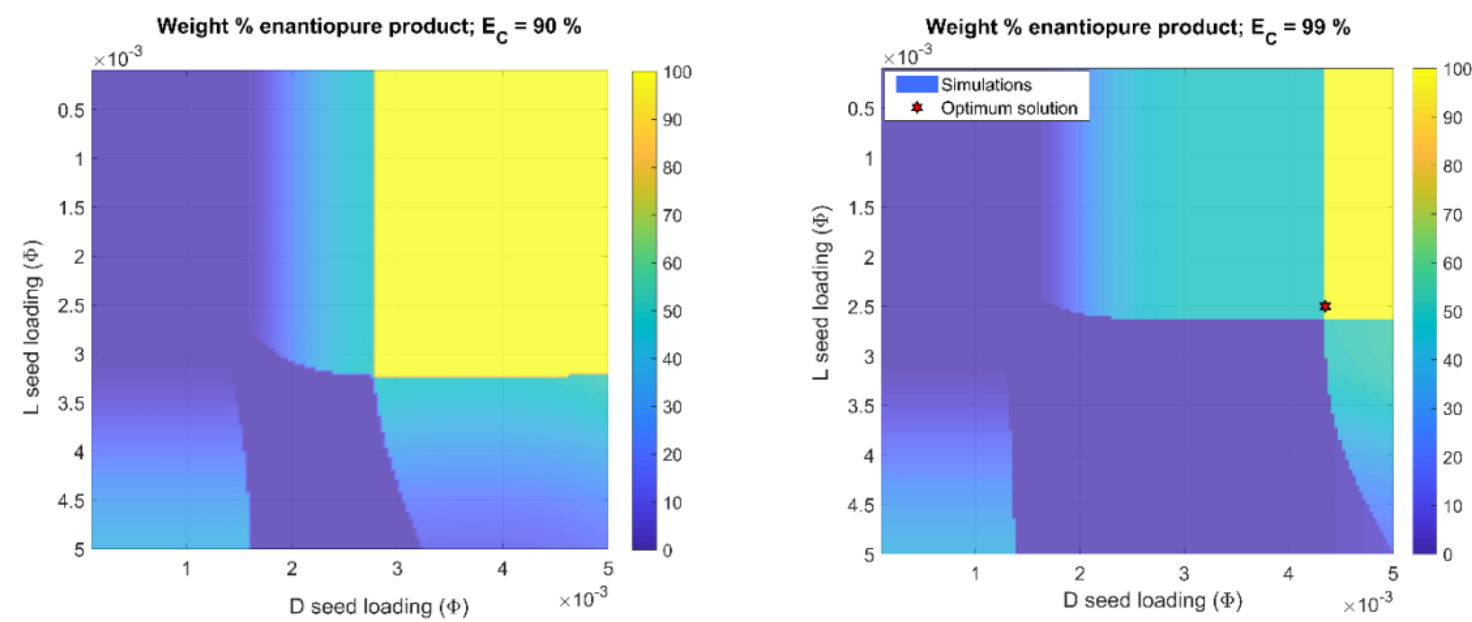

Figure 5. Achievable separation performance by sieving as a function of enantiomer seed loadings. The simulation conditions for both $E_{c}=90 \%$ and $E_{c}=99 \%$ are shown in Table 4: $L_{-}$ asn seed distribution $=\mathrm{CSD} 4, \mathrm{D}$-asn seed distribution $=\mathrm{CSD} 3$, batch cooling time $=5$-hour, linear cooling from 41.6 to $20^{\circ} \mathrm{C}$.

\subsection{Concomitant Crystallization Experiments}

The input seed CSDs and seed volume fractions, as well as the optimum cooling temperature profile (3-hour and 5-hour cooling time with a 2-hour equilibration time) determined in the optimization simulations were carried forward for experimental demonstration. Experimental seed CSD results obtained from the Mastersizer are shown in Figure 6. The CSD measurements showed that distributions of L-asn and D-asn were similar for the sieve fractions for which material for both enantiomers was available. No D-asn was available for the two smallest sieve fractions. In the experiments, therefore, all seed classes 1-8 were available for L-asn and seed classes 3-8 were available for D-asn. In the experiments we chose to work with 3 and 5-hour cooling times with a 2-hour equilibration time. 


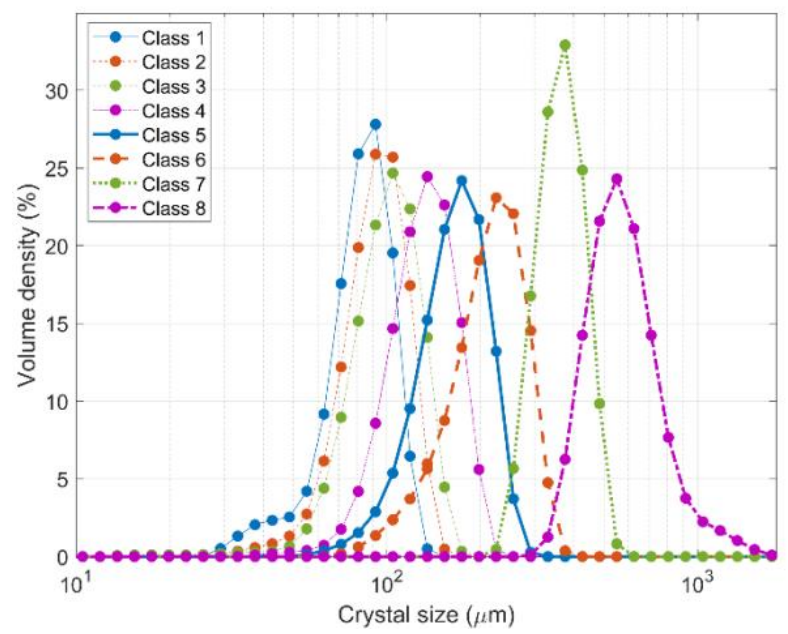

Figure 6. Seed crystal size distributions used in the experimental concomitant crystallization experiments determined by the Malvern Mastersizer. All seed classes 1-8 were available for Lasn and seed classes 3-8 were available for D-asn (we assume that D-asn seeds have the same CSD as the L-asn seeds). The continuous lines are a guide to the eye.

A total of five experiments were carried out, the details of which are given in Table 2. Figure 7 shows the results of each experiment where the mass fraction $X_{\mathrm{i}}(L)$ and $X_{\mathrm{i}}(D)$ of enantiomer in respect to the total product mass is shown for each product sieve fraction and, within each sieve fraction, the enantiomeric excess $E$. If product collected in a sieve fraction has an enantiomeric excess of less than $80 \%$ then that material is considered to be impure. This purity threshold is also shown in each plot in Figure 7a-e for both L-asn (+80\%) and D-asn (-80\%).

In experiment 1 (Figure 7a) 100\% D-asn product was collected in both the 355-500 $\mu \mathrm{m}$ and $>500 \mu \mathrm{m}$ sieve fractions. Some crystals of D-asn were found in sieve fractions $125-180 \mu \mathrm{m}$ and 180-250 $\mu \mathrm{m}$, however there was still an enantiomeric excess $>80 \%$ with respect to L-asn product in those sieve fractions. Pure L-asn product was collected in the 90-125 $\mu \mathrm{m}$ sieve fraction and below. Impure product was collected only in the $250-355 \mu \mathrm{m}$ sieve fraction meaning only $8.1 \%$ of the total product mass collected was impure.

A similar result was obtained for experiment 3, where only $6.0 \%$ of the total product mass collected was impure (product in sieve fractions 20-45 $\mu \mathrm{m}, 45-63 \mu \mathrm{m}$ and 180-250 $\mu \mathrm{m}$ ). In experiments 2 and 5 (Figure $7 \mathrm{~b}$ and 7d, respectively), the percentage of impure product collected was $16.5 \%$ and $14.4 \%$ of the total product mass, respectively. Of the five experiments carried out in Table 2, only one experiment produced more than $20 \mathrm{wt} \%$ impure product; in experiment $4,55.9 \%$ of the total product mass collected was impure. 
Figure 7 shows that in all the experiments a small fraction of product has been collected in the sieve fraction lower than the lowest input seed size (with the exception of experiment 2, where product has been collected in the sieve fraction equal to the seed input size). This observation gives a strong indication that secondary nucleation is taking place in the crystallizer. Evidence that secondary nucleation occurred is also observed in the FBRM count (Figure 8) and chord length distribution (CLD) trends (Figure 9) from each experiment.
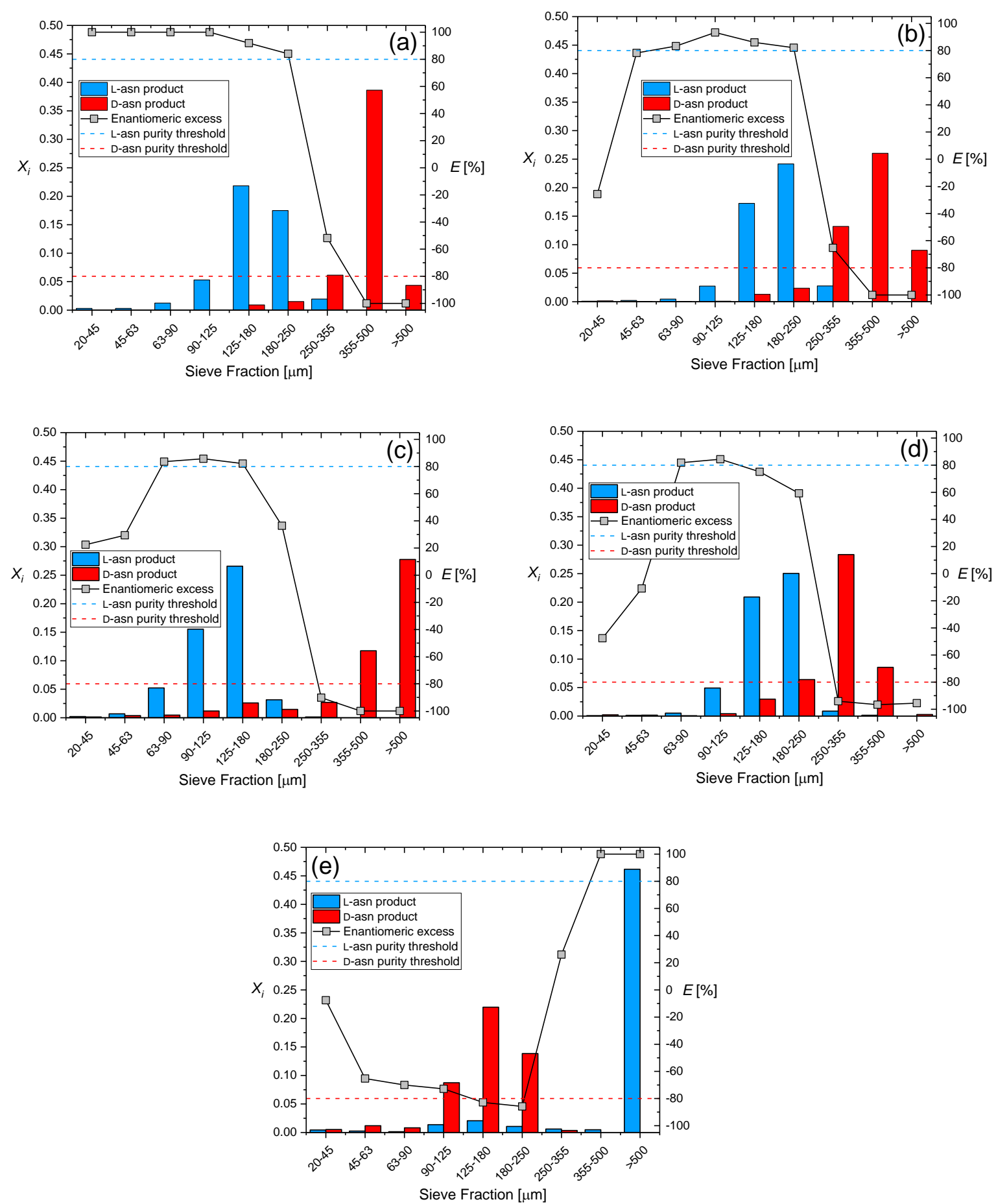
Figure 7. Product CSDs for L-asn ( $\square$ ) and D-asn ( $\square$ ) crystals collected via sieving after filtration washing and drying for experiments 1-5 (a-e, respectively) in Table 2. Each sieve column shows the mass fraction $X_{i}$ of total product obtained for L-asn and D-asn as well as the enantiomeric excess (-m-) of product in each sieve fraction. A purity threshold is set for both L-asn (--) at $+80 \%$ enantiomeric excess and D-asn (--) at $-80 \%$ enantiomeric excess.

Each plot in Figure 8a-e shows the FBRM count trends and cooling profiles for experiment 15. Count trends are shown for the corresponding sieve fractions available: 45-63, 63-90, 90$125,125-180$ and $180-250 \mu \mathrm{m}$. FBRM is a useful PAT tool than can give a good indication of the mechanisms occurring in the process e.g. crystal growth and nucleation. However, it cannot give accurate size information since, for example, a 10-micron chord length might have been measured at the edge of a 100-micron crystal.

In the FBRM count trends in Figure 8a, a sharp increase in the number of counts for each sieve fraction chord length bin can be seen at around $20 \mathrm{~min}$; this is the point at which seed crystals were added to the crystallizer (see Table 2 for seed loading details). After seeding, there is a decrease in the number of counts for chord lengths $45-63 \mu \mathrm{m}$ and 63-90 $\mu \mathrm{m}$. This could indicate growth of the seed crystals since, at the same time, we see an increase in the number of counts for 90-125 $\mu \mathrm{m}, 125-180 \mu \mathrm{m}$ and 180-250 $\mu \mathrm{m}$. Over time in each experiment we see continued increase in the number of counts for 90-125 $\mu \mathrm{m} 125-180 \mu \mathrm{m}$ and 180-250 $\mu \mathrm{m}$ chord lengths. However, there is also an increase in the number of counts for chord lengths 45-63 $\mu \mathrm{m}$ and 63-90 $\mu \mathrm{m}$. This would indicate that the number of smaller particles in the crystallizer is increasing, most likely due to secondary nucleation in the system. Similar trends can be seen in experiments 2-5 (Figure 8b-e, respectively).

For each plot in Figure 9a-e, the seed CLD is shown (black dashed line) as well as CLDs are various time intervals throughout each experiment. For experiments with a 5-hour cooling profile, CLDs are shown at 1-hour intervals. For experiments with a 3-hour cooling profile, CLDs are shown at 30 min intervals. In each experiment 1-5 (Figure 9a-e, respectively) there is an overall trend in each experiment with time that the CLD shifts to the right, indicating crystal growth during the process. However, the inset of each plot on Figure $9 \mathrm{a}-\mathrm{d}$ expands the region of the each CLD front. 

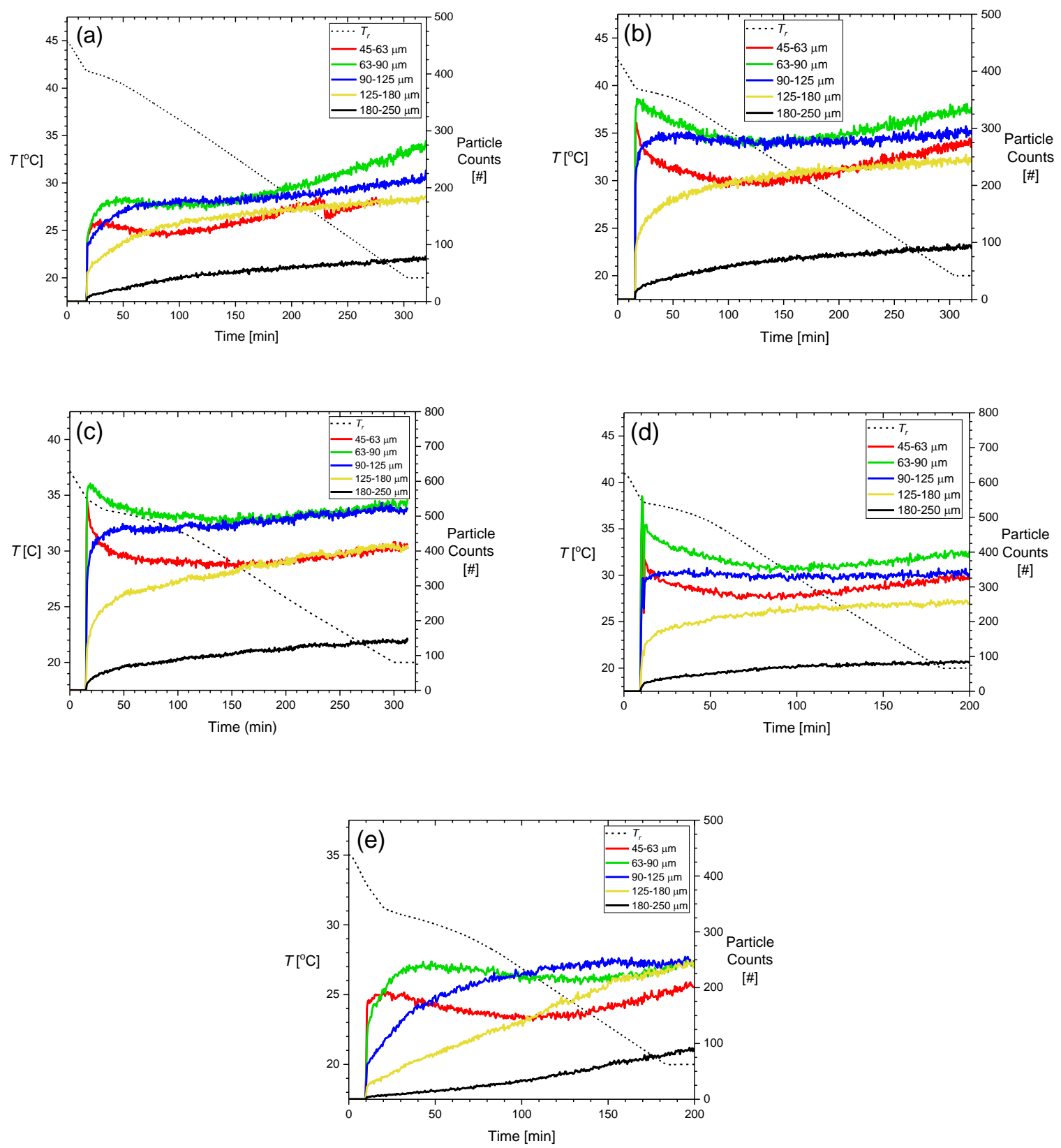

Figure 8. Temperature and FBRM count trends for experiments 1-5 (a-e, respectively) in Table 2. Each plot shows $T_{r}=$ reactor temperature (--) as a function of time and the measured particle

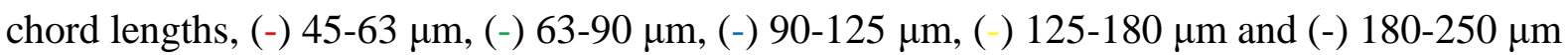
as a function of time.

In experiment 1 (Figure 9a), whilst there is an overall shift of the whole CLD to the right, we see that over time the CLD front shifts to the left with each sample point. This indicates that over time there are also more and more small particles being produced; another indication of secondary nucleation. In Figure 9b-e (experiments 2-5, respectively), there is an initial shift to the right of the CLD front (following the trend of the overall CLD) meaning that initially only 
growth is occurring. Then the CLD front starts trending to the left at $180 \mathrm{~min}$ in experiment 2 (Figure 9b), $240 \mathrm{~min}$ in experiment 3 (Figure 9c) and $120 \mathrm{~min}$ in experiment 4 (Figure 9d) and $150 \mathrm{~min}$ in experiment 5 (Figure 9e) indicating that secondary nucleation has started to occur in each of these experiments.
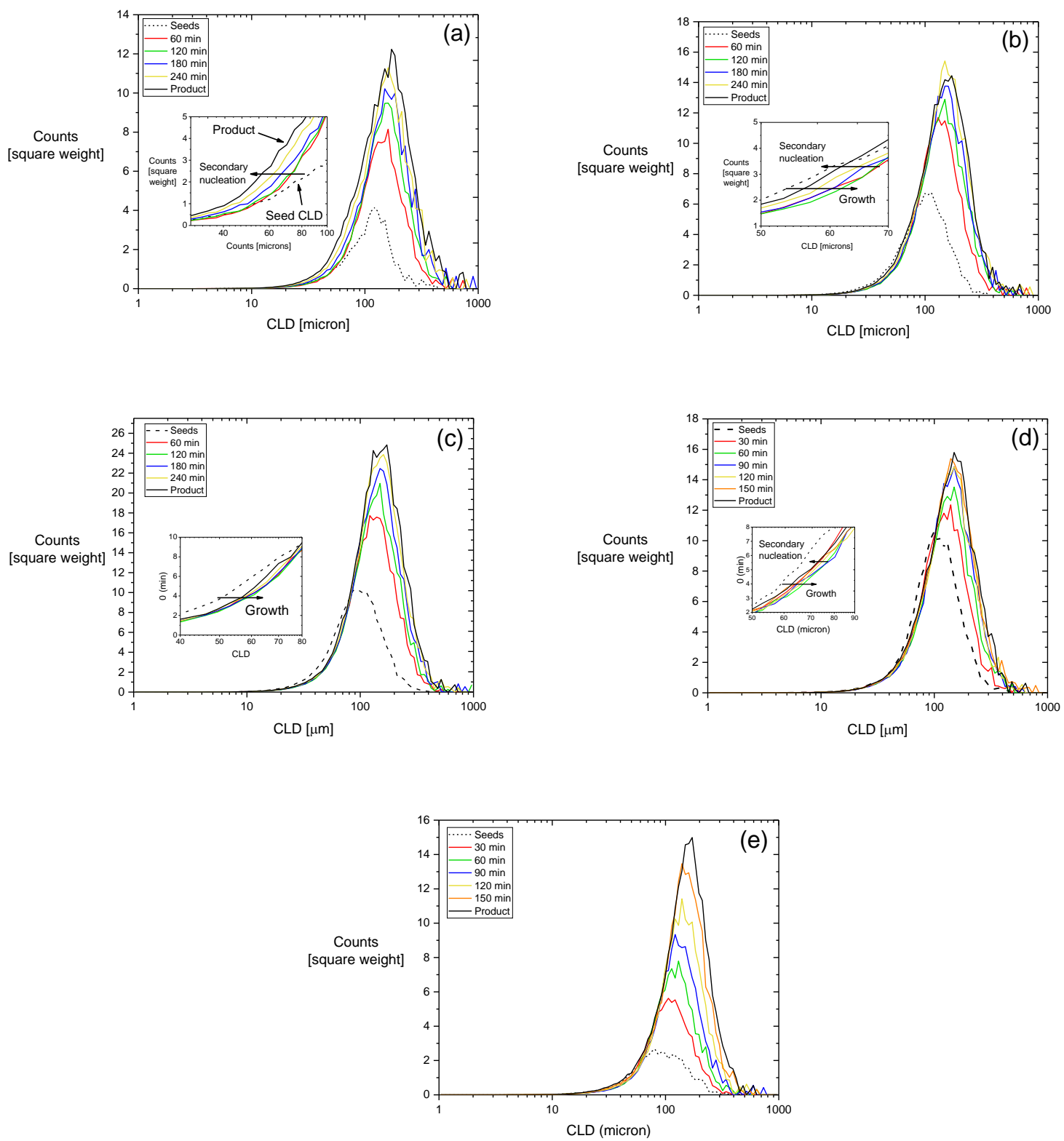

Figure 9. Chord length distribution (CLD) trends for experiments 1-5 (a-e, respectively) in Table 2. For experiments with a 5 hour cooling profile (experiments 1-3) the measured CLD at 60 min intervals in plot a-c: (--) seed (0 min), (-) $60 \mathrm{~min},(-) 120 \mathrm{~min},(-) 180 \mathrm{~min}$, (-) $^{2} 40 \mathrm{~min}$ and (-) product. For experiments with a 3 hour cooling profile (experiments 4 and 5) the measured CLD at $30 \mathrm{~min}$ intervals is shown in plots $\mathrm{d}$ and e: (--) seed (0 min), (-) $30 \mathrm{~min},(-)$ $60 \mathrm{~min},(-) 90 \mathrm{~min},(-) 120 \mathrm{~min},(-) 150 \mathrm{~min}$ and (-) product. The inset of each plot highlight 
any shifts in the CLD trend for each experiment that indicate any potential secondary nucleation.

Additionally, PVM images of experiments 1-5 in Table 2, as shown in Figure 10, show images of the suspension just after seeding and just before product was collected are shown. The images show the formation of some much smaller particles in the product compared to the seed crystals added (these particles are indicated by a red circles). This gives further indication of secondary nucleation processes occurring during the process. Note that no image analysis was conducted on the PVM images with regards to crystal shape during the process. The PVM probe was simply used to complement the FBRM probe with respect to nucleation, agglomeration or breakage of the crystals.
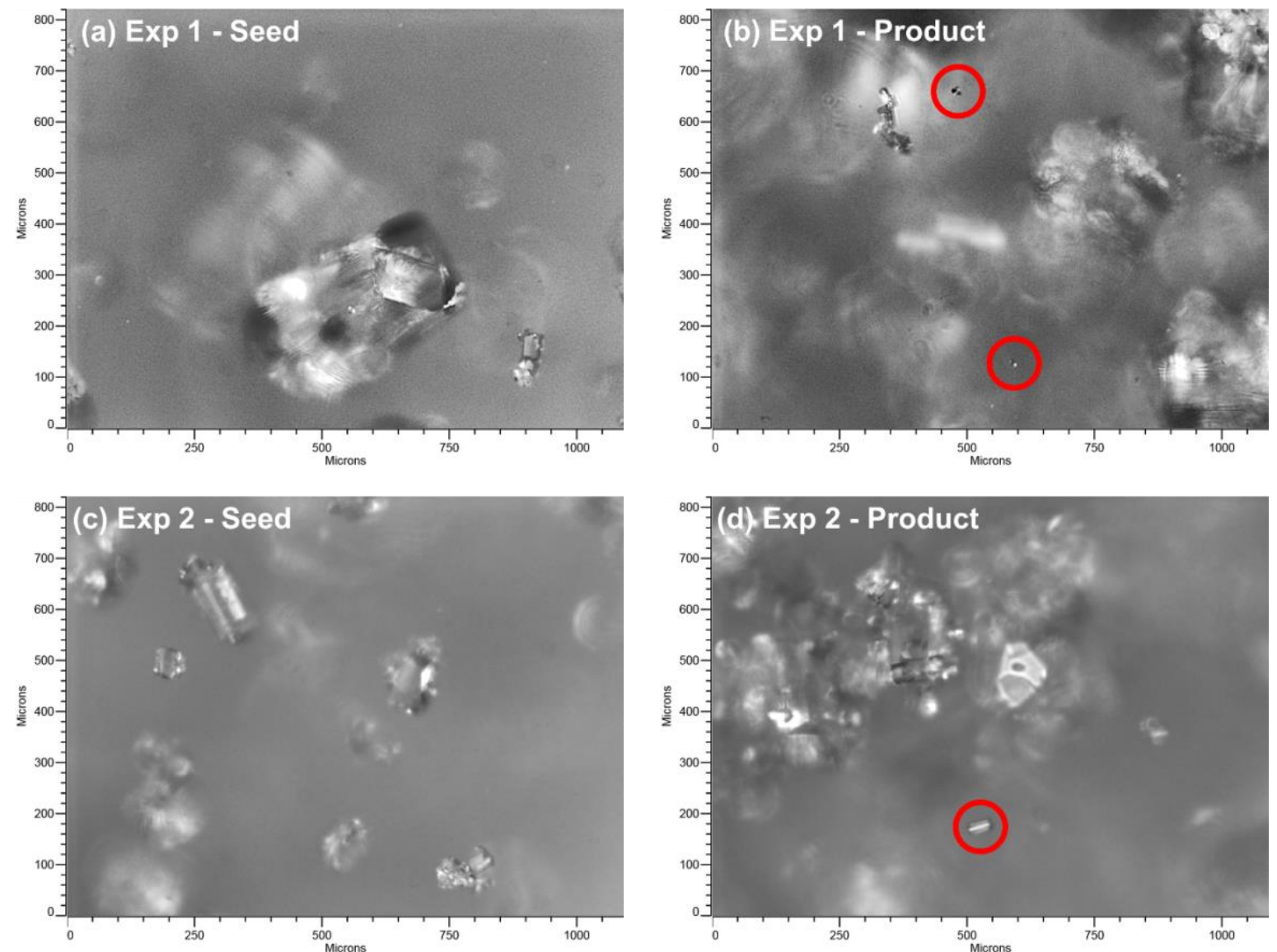

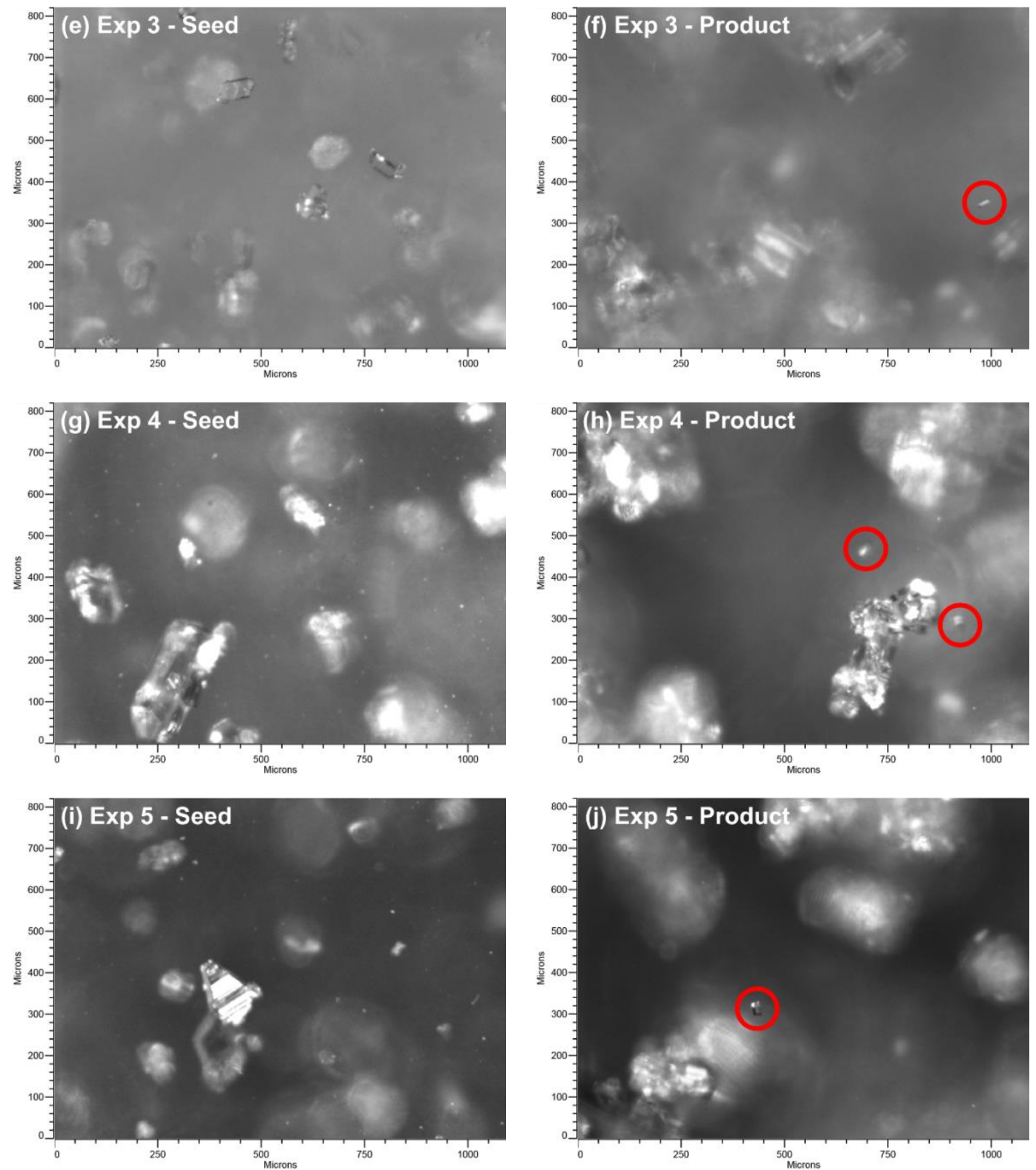

Figure 10. PVM images from experiments 1-5 in Table 2 showing the suspension just after seeding (left) and just before product collection (right). The red circles highlight the formation of small particles during the process, most likely due to secondary nucleation.

The effect of secondary nucleation can also be seen in the average product crystal sizes obtained from each enantiomer and comparing this value to a predicted average crystal size expected to be obtained. Assuming the number of seeds is equal to the number of product crystals (no nucleation or agglomeration) a product size can be determined with Eq. (24): ${ }^{35}$ 
$L_{p}^{3}=L_{s}^{3} \times \frac{\Delta m+m_{s}}{m_{s}}$

$L_{p}$ is the average size of the product crystals $p, L_{s}$ is the average size of the seed crystals, $\Delta m$ is the change in mass due to crystallization and $m_{s}$ is the mass of seeds added at the start of the crystallization. Use of this equation further assumes an invariable shape of asparagine monohydrate crystals. ${ }^{33}$ Figure 11 shows the obtained average crystal size versus predicted average crystal size. The straight line through the origin indicates that the predicted size is equal to the experimental size of the product for a point which lies on that line. Points which lie below this line indicate that the predicted average crystal size is greater than experimentally obtained average crystal size, meaning that it is likely that crystals have been formed during the process by means of secondary nucleation or breakage. Points which lie above this line indicate that the obtained average crystal size is larger than the predicted average crystal size, indicating that the number of crystals has decreased during the process, most likely due to agglomeration of crystals. The obtained average crystal size of each enantiomer $\bar{x}_{C S D}^{(e)}$ was calculated by:

$\bar{x}_{C S D}^{(e)}=2 \sum X_{i}^{(e)} \bar{x}_{F}$

where $X_{i}^{(e)}$ is the mass fraction of enantiomer $e$ in sieve fraction $F$ and $\bar{x}_{F}$ is the average size of sieve fraction $F$ assuming a normal distribution within the sieve fraction. The sum is multiplied by 2 since each enantiomer makes up only $50 \%$ of the product. Figure 11 shows that in all experiments, crystals of D-asn undergo secondary nucleation. In experiments 2 and 3, Lasn crystals are shown to undergo ideal growth. However from the separation plots in Figure $7 \mathrm{~b}$ and $6 \mathrm{c}$, respectively, we can see that some secondary nucleation of L-asn does occur. Figure 11 also shows that for experiment 4 some agglomeration of L-asn crystals may have occurred since the obtained average product crystal size is larger than the predicted average crystal size in this experiment. 


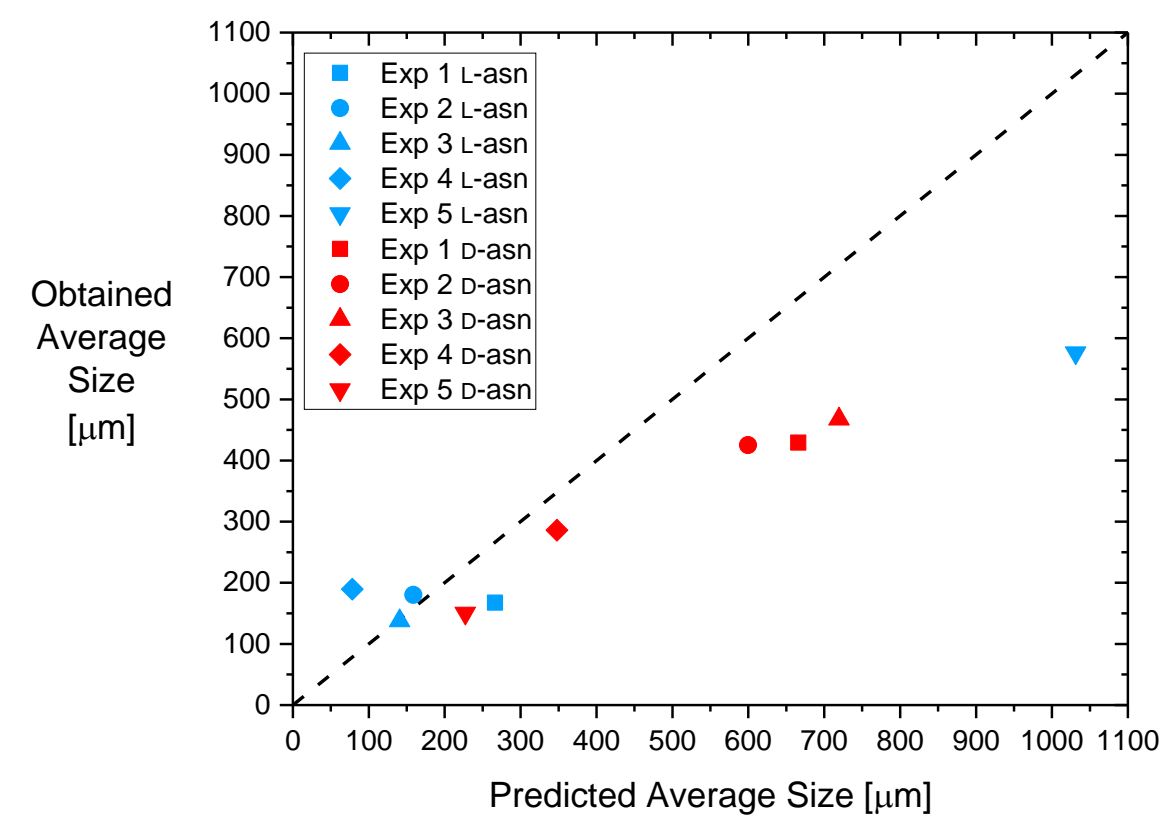

Figure 11. Obtained versus predicted average product particle size of both L-asn and D-asn crystals from the separation experiments carried out in Table 2: L-asn (blue) and D-asn (red) average crystal sizes for experiment $1(\square, \varpi)$, experiment $2(\bullet, \bullet)$, experiment $3(\Delta, \Delta)$, experiment $4(\diamond, \diamond)$ and experiment $5(\nabla, \nabla)$. The straight line through the origin indicates the predicted average crystal size is equal to the experimentally obtained average crystal size. Points which show the predicted size is greater than the obtained size indicate formation of crystals during the process by secondary nucleation.

\section{DISCUSSION}

The majority of sieve fractions possess an enantiomeric excess above the threshold value of 99\%. As many of the other sieve fractions still contain a substantial enantiomeric excess, these can be used in subsequent conventional preferential crystallization processess. As long as the enantiomeric excess achieved in a sieve fraction is large enough to begin with, this subsequent preferential crystallization process would be carried out in the biphasic domain of the phase diagram, the compositional region where enantiopure material can be obtained. In this way an enantiomeric excess of commercial significance could be achieved also for these sieve fractions. Then, the next objective to achieve is to increase productivity (g product / liter solvent in time) and yield (fraction of potential product crystallized). For this, the cooling profile and final crystallization temperature of the process can be further optimized to maximize the productivity and yield of the process (since, for this work, for example, the final crystallization temperature used was based on previous work ${ }^{16}$ ). 
Whilst the model-based optimization generates the optimal process conditions (initial seed CSDs and seed loadings of both enantiomers as well as initial temperature and temperature cooling profile) to achieve chiral separation through sieving, experimentally, the actual separation efficiency achieved depends on several additional factors. First, it is important to control a constant number of crystals during the process i.e. by minimizing nucleation or agglomeration, so that only growth of the crystals occurs and therefore separation of the CSDs at the end of the process yields enantiopure material. Therefore, great care was taken to control crystallization conditions such as supersaturation during the process. Although the simulations suggest conditions to avoid secondary nucleation, this experimentally still may occur, compromising the CSD of both enantiomers. Also seed quality can have a profound effect on the crystallization outcome. In this work, seeds were prepared by sieving the raw material obtained from the supplier. No further pre-treatment of the seeds was carried out. Therefore, it is possible that small fines could exist on the surface of the seeds. These fines can become dislodged during mixing and act as secondary nuclei. ${ }^{36,37}$ This is known as initial breeding. ${ }^{38}$ Furthermore, if any of the pre-sieved seed crystals are aggregated (meaning they would not pass through the sieve during seed preparation), they could become de-aggregate by fluid mixing in the crystallizer during the process. However, this mechanism is least likely to occur.

Furthermore, it is important to consider in the model all the crystallization mechanisms that could be occurring during the process. The optimization model developed incorporates growth of the seed crystals only. Therefore, no agglomeration, breakage, primary or secondary nucleation mechanisms are taken into consideration as they are assumed to be negligible. In reality, however, these mechanisms may occur (probably with the exception of primary nucleation since we remain close to the solubility curve during cooling and therefore far away from the metastable limit). Since agglomeration, breakage and secondary nucleation will most probably occur, they are likely to affect the separation results obtained. For instance, secondary nuclei from larger crystals of one enantiomer will be collected in a smaller sieve fraction containing crystals of the opposite enantiomer, as was observed in experiments 2-5. Additionally, with the inclusion of nucleation terms, dissolution terms can be added to the optimization. This would allow for the design of similar processes but with temperature cycling, which would increase the flexibility of the process significantly while also improving process productivity and yield. In future work, therefore, it may be useful to also incorporate each of these crystallization mechanisms (secondary nucleation, agglomeration, breakage) into the optimization model before conducting experiments. Another factor that can play an important role is growth rate dispersion. It is common to observe growth rates in the population 
ranging between 0.2-5 times the average growth rate. If this happens, it causes broadening of the CSDs resulting in wider overlaps, leading to central sieve fraction being mixtures of the two enantiomers. To alleviate the reduced purity in the product, the mixture sieve fractions can be re-dissolved and re-crystallized. However, incorporating growth rate dispersion into the model allows an improved prediction of the bias in the CSDs needed to come to increased purity in the sieve fractions. It should be considered, however, that the more complex modelbased optimization will take significantly more computational time for the design of the crystallization conditions. However, this work has shown that even by using a simple growth only model-based two-level optimization it is possible to obtain excellent separation results and, therefore addition of these other crystallization mechanisms may not be required.

Finally, the results for experiment 4 , however, seem to suggest that L-asn product crystals underwent some agglomeration since the obtained average crystal size in the L-asn sieve fraction was higher than the predicted average crystal size. This is likely due to any variances that were experienced during the filtration, washing and drying stage of the process since agglomeration was not observed in any other experiments in Table 2. These variances could be due to differences in drying time and conditions since product was only left to dry in a vacuum oven overnight and not for a specific length of time. Additionally, breakage of crystals can also occur during sieving, and since the shape of the asparagine crystals is far from spherical, the separation results via sieving could be affected. However, since the actual measured CSD of each seed sieve fraction was used as input for the optimization model, these errors should be minimized. For further optimization of this type of separation process, it is important to consider each of these aforementioned factors. Of course, depending on the compound of interest, each factor may be more or less significant than observed in this work.

\section{CONCLUSIONS}

We have demonstrated a novel separation strategy for enantiomers of a conglomerate forming compound via mechanical separation by sieving. Through the use of a digital design strategy, applying a two-level optimization-based design using a population balance model of the system, experimental conditions such as seed size and loading of each enantiomer and the temperature profile were optimized to achieve the separation of enantiomers based on their product crystal size. Although the model considers crystal growth only and assumes the absence of nucleation (primary or secondary), agglomeration or breakage, it has been shown to be effective in providing optimized crystallization conditions. Through this process, 
unwanted primary nucleation is minimized since the system is seeded with both enantiomers and through the optimized cooling profile, the operating trajectories remain close to the solubility curve. For even better separation results using this approach the population balance model could be refined to include other crystallization mechanisms such as nucleation (primary and secondary) as well as agglomeration, breakage, and growth rate dispersion. In general, these other mechanisms may also occur, as the experimental results presented here also indicated some secondary nucleation. Furthermore, it was found that the filtration, washing and drying procedures for the product may have significant effect in the success or failure of this separation process. Therefore, this should also be investigated and optimized for the best separation results.

\section{SUPPORTING INFORMATION}

Secondary nucleation curve determination.

\section{ACKNOWLEDGEMENTS}

ASD would like to thank the Nagy group at Purdue Univeristy, IN, USA, for enjoying the hospitality that he enjoyed during his 3 month research visit on the topic of this paper. We would like to thank the EPSRC and the Doctoral Training Centre in Continuous Manufacturing and Crystallisation (Grant Ref: EP/K503289/1) for funding this work. The authors would like to acknowledge that the experimental work was carried out in the CMAC National Facility supported by UKRPIF (UK Research Partnership Fund) award from the Higher Education Funding Council for England (HEFCE) (Grant ref HH13054). The authors would also like to acknowledge the International Fine Particles Research Institute (IFPRI) for partial financial support (Grant No. FRR-21-09).

\section{REFERENCES}

(1) Srisanga, S.; ter Horst, J. H. Racemic Compound, Conglomerate, or Solid Solution: Phase Diagram Screening of Chiral Compounds. Cryst. Growth Des. 2010, 10 (4), 18081812. https://doi.org/10.1021/cg901483v.

(2) Nguyen, L. A.; He, H.; Pham-Huy, C. Chiral Drugs: An Overview. Int. J. Biomed. Sci. 2006, 2 (2), 85-100.

(3) Curitiba Marcellos, C. F.; Durand, H.; Kwon, J. S.-I.; Gomes Barreto, A.; Lage, P. L. da C.; Bezerra de Souza, M.; Secchi, A. R.; Christofides, P. D. Optimal Operation of Batch 
Enantiomer Crystallization: From Ternary Diagrams to Predictive Control. AIChE J. 2017. https://doi.org/10.1002/aic.16028.

(4) Spix, L.; Alfring, A.; Meekes, H.; Van Enckevort, W. J. P.; Vlieg, E. Formation of a Salt Enables Complete Deracemization of a Racemic Compound through Viedma Ripening. https://doi.org/10.1021/cg4018882.

(5) Li, W. W.; Spix, L.; de Reus, S. C. A.; Meekes, H.; Kramer, H. J. M.; Vlieg, E.; ter Horst, J. H. Deracemization of a Racemic Compound via Its Conglomerate-Forming Salt Using Temperature Cycling. Cryst. Growth Des. 2016, 16 (9), 5563-5570. https://doi.org/10.1021/acs.cgd.6b01034.

(6) Spix, L.; van Enckevort, W. J. P.; van der Wal, L. J. M.; Meekes, H.; Vlieg, E. Resolution of Asparagine in a Coupled Batch Grinding Process: Experiments and Modelling. $\quad$ CrystEngComm 2016, $18 \quad$ (48), 9252-9259. https://doi.org/10.1039/C6CE02043G.

(7) Xie, S.-M.; Yuan, L.-M. Recent Progress of Chiral Stationary Phases for Separation of Enantiomers in Gas Chromatography. J. Sep. Sci. 2017, 40 (1), 124-137. https://doi.org/10.1002/jssc.201600808.

(8) Huang, X.-Y.; Di, D.-L. Chiral Separation by Counter-Current Chromatography. TrAC Trends Anal. Chem. 2015, 67, 128-133. https://doi.org/10.1016/J.TRAC.2015.01.009.

(9) Patel, D. C.; Wahab, M. F.; Armstrong, D. W.; Breitbach, Z. S. Advances in HighThroughput and High-Efficiency Chiral Liquid Chromatographic Separations. J. Chromatogr. A 2016, 1467, 2-18. https://doi.org/10.1016/J.CHROMA.2016.07.040.

(10) Jacques, J.; Collet, A. Enantiomers, Racemates and Resolutions; John Wiley \& Sons Ltd, 1981.

(11) Levilain, G.; Coquerel, G. Pitfalls and Rewards of Preferential Crystallization. CrystEngComm 2010, 12 (7), 1983. https://doi.org/10.1039/c001895c.

(12) Elsner, M. P.; Ziomek, G.; Seidel-Morgenstern, A. Efficient Separation of Enantiomers by Preferential Crystallization in Two Coupled Vessels. AIChE J. 2009, 55 (3), 640649. https://doi.org/10.1002/aic.11719.

(13) Chaaban, J. H.; Dam-Johansen, K.; Skovby, T.; Kiil, S. Separation of Enantiomers by Continuous Preferential Crystallization: Experimental Realization Using a Coupled Crystallizer Configuration. Org. Process Res. Dev. 2013, 17 (8), 1010-1020. https://doi.org/10.1021/op400087g.

(14) Elsner, M. P.; Ziomek, G.; Seidel-Morgenstern, A. Simultaneous Preferential Crystallization in a Coupled Batch Operation Mode. Part II: Experimental Study and 
Model Refinement. Chem. Eng. Sci. 2011, 66 (6), 1269-1284. https://doi.org/10.1016/j.ces.2010.12.035.

(15) Galan, K.; Eicke, M. J.; Elsner, M. P.; Lorenz, H.; Seidel-Morgenstern, A. Continuous Preferential Crystallization of Chiral Molecules in Single and Coupled MixedSuspension Mixed-Product-Removal Crystallizers. Cryst. Growth Des. 2015, 15 (4), 1808-1818. https://doi.org/10.1021/cg501854g.

(16) Dunn, A. S.; Svoboda, V.; Sefcik, J.; ter Horst, J. H. Resolution Control in a Continuous Preferential Crystallization Process. Org. Process Res. Dev. 2019, 23 (9), 2031-2041. https://doi.org/10.1021/acs.oprd.9b00275.

(17) Majumder, A.; Nagy, Z. A Comparative Study of Coupled Preferential Crystallizers for the Efficient Resolution of Conglomerate-Forming Enantiomers. Pharmaceutics 2017, 9 (4), 55. https://doi.org/10.3390/pharmaceutics9040055.

(18) Kongsamai, P.; Maneedaeng, A.; Flood, C.; ter Horst, J. H.; Flood, A. E. Effect of Additives on the Preferential Crystallization of L-Asparagine Monohydrate. Eur. Phys. J. Spec. Top. 2017, 226 (5), 823-835. https://doi.org/10.1140/epjst/e2016-60257-3.

(19) Mastai, Y.; Völkel, A.; Cölfen, H. Separation of Racemate from Excess Enantiomer of Chiral Nonracemic Compounds via Density Gradient Ultracentrifugation. J. AM. CHEM. SOC 2008, 130, 2426-2427. https://doi.org/10.1021/ja067905i.

(20) Zhang, S.; Huang, Y.; Zhou, L.; Yang, Y.; Xie, C.; Wang, Z.; Hou, B.; Hao, H.; Bao, Y.; Yin, Q. Novel Technology for Separation of Binary Eutectic-Forming Mixture by Cocrystallization into Different Sizes Combined with Particle Size Fraction. Ind. Eng. Chem. Res. 2019, acs.iecr.9b01293. https://doi.org/10.1021/acs.iecr.9b01293.

(21) Norihito Doki, *; Masaaki Yokota; Shigeko Sasaki, and; Kubota, N. Simultaneous Crystallization of D- and 1-Asparagines in the Presence of a Tailor-Made Additive by Natural Cooling Combined with Pulse Heating. 2004. https://doi.org/10.1021/CG049758A.

(22) Maillard, D.; Koller, G.; Wakaresko, E.; Gottburg-Reininger, S. Process of Isolating Enantiomer Components from Enantiomer Mixtures by Particle-Size- Controlled Crystallization. US8846911B2, September 30, 2014.

(23) Petruševska-Seebach, K.; Seidel-Morgenstern, A.; Elsner, M. P. Preferential Crystallization of L-Asparagine in Water. Cryst. Growth Des. 2011, 11 (6), 2149-2163. https://doi.org/10.1021/cg101408e.

(24) Randolph, A. D.; Larson, M. A. Theory of Particulate Processes, Second.; Academic Press, Inc.: San Diego, 1988. 
(25) Wright, D. L.; McGraw, R.; Rosner, D. E. Bivariate Extension of the Quadrature Method of Moments for Modeling Simultaneous Coagulation and Sintering of Particle Populations. J. Colloid Interface Sci. 2001, $236 \quad$ (2), 242-251. https://doi.org/10.1006/jcis.2000.7409.

(26) Aamir, E.; Nagy, Z. K.; Rielly, C. D.; Kleinert, T.; Judat, B. Combined Quadrature Method of Moments and Method of Characteristics Approach for Efficient Solution of Population Balance Models for Dynamic Modeling and Crystal Size Distribution Control of Crystallization Processes. Ind. Eng. Chem. Res. 2009, 48 (18), 8575-8584. https://doi.org/10.1021/ie900430t.

(27) Mesbah, A.; Kramer, H. J. M.; Huesman, A. E. M.; Van den Hof, P. M. J. A Control Oriented Study on the Numerical Solution of the Population Balance Equation for Crystallization Processes. Chem. Eng. Sci. 2009, 64 (20), 4262-4277. https://doi.org/10.1016/J.CES.2009.06.060.

(28) Omar, H. M.; Rohani, S. Crystal Population Balance Formulation and Solution Methods: A Review. Cryst. Growth Des. 2017, $17 \quad$ (7), 4028-4041. https://doi.org/10.1021/acs.cgd.7b00645.

(29) Porru, M.; Özkan, L. Monitoring of Batch Industrial Crystallization with Growth, Nucleation, and Agglomeration. Part 1: Modeling with Method of Characteristics. Ind. Eng. Chem. Res. 2017, 56 (20), 5980-5992. https://doi.org/10.1021/acs.iecr.7b00240.

(30) Aamir, E.; Nagy, Z. K.; Rielly, C. D. Optimal Seed Recipe Design for Crystal Size Distribution Control for Batch Cooling Crystallisation Processes. Chem. Eng. Sci. 2010, 65 (11), 3602-3614. https://doi.org/10.1016/J.CES.2010.02.051.

(31) Nagy, Z. K.; Aamir, E. Systematic Design of Supersaturation Controlled Crystallization Processes for Shaping the Crystal Size Distribution Using an Analytical Estimator. Chem. Eng. Sci. 2012, 84, 656-670. https://doi.org/10.1016/j.ces.2012.08.048.

(32) Shahzad, A.; Kerrigan, E. C.; Constantinides, G. A. A Warm-Start Interior-Point Method for Predictive Control. In IET Seminar Digest; 2010; Vol. 2010, pp 949-954. https://doi.org/10.1049/ic.2010.0409.

(33) Temmel, E.; Gänsch, J.; Lorenz, H.; Seidel-Morgenstern, A. Measurement and Evaluation of the Crystallization Kinetics of 1 -Asparagine Monohydrate in the Ternary 1 -/ d -Asparagine/Water System. Cryst. Growth Des. 2018, 18 (12), 7504-7517. https://doi.org/10.1021/acs.cgd.8b01322.

(34) Choong, K. L.; Smith, R. Optimization of Batch Cooling Crystallization. Chem. Eng. Sci. 2004, 59 (2), 313-327. https://doi.org/10.1016/j.ces.2003.09.025. 
(35) Mullin, J. W. Crystallization, Fourth Edi.; Butterworth-Heinemann, 2001.

(36) Cruz, P.; Rocha, F.; Ferreira, A. Determination of the Critical Mixing Intensity for Secondary Nucleation of Paracetamol in an Oscillatory Flow Crystallizer. CrystEngComm 2018, 20 (6), 829-836. https://doi.org/10.1039/C7CE01940H.

(37) Frawley, P. J.; Mitchell, N. A.; Ó'Ciardhá, C. T.; Hutton, K. W. The Effects of Supersaturation, Temperature, Agitation and Seed Surface Area on the Secondary Nucleation of Paracetamol in Ethanol Solutions. Chem. Eng. Sci. 2012. https://doi.org/10.1016/j.ces.2012.03.041.

(38) Steendam, R. R. E.; Frawley, P. J. Secondary Nucleation of Sodium Chlorate: The Role of Initial Breeding. Cryst. Growth Des. 2019, acs.cgd.9b00317. https://doi.org/10.1021/acs.cgd.9b00317. 


\section{FOR TABLE OF CONTENTS USE ONLY}

Enabling Mechanical Separation of Enantiomers through Controlled Batch-wise Concomitant Crystallization: Digital Design and Experimental Validation

Andrew S. Dunn ${ }^{1,2}$, Botond Szilagyi ${ }^{3}$, Joop H. ter Horst ${ }^{2,4, *}$, Zoltan K. Nagy ${ }^{3,5, *}$

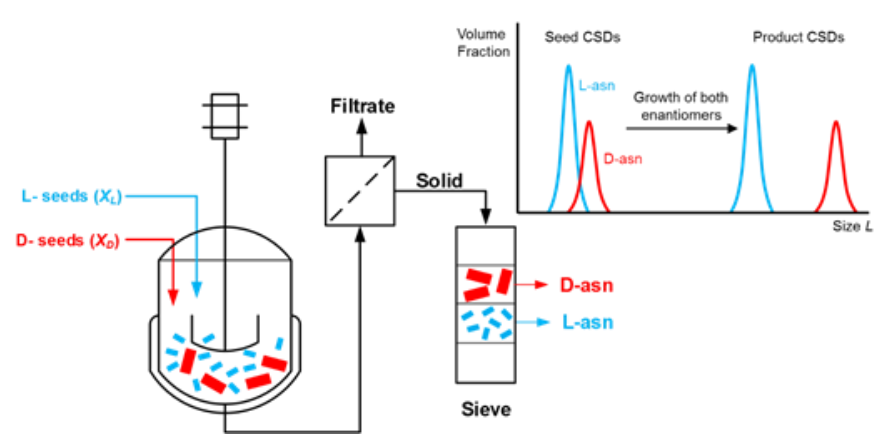

Synopsis: Different seed sizes of each enantiomer are added to the crystallizer, such that they both grow simultaneously and can be mechanically separated by their product crystal size distribution by sieving at the end of the crystallization process. 